\title{
Multi-Label Classification based on Sum-Product Networks
}

\author{
Julissa Giuliana Villanueva Llerena
}

\author{
DisSERTAÇÃO APRESENTADA \\ $\mathrm{AO}$ \\ Instituto de MatemáticA e EstatísticA \\ DA \\ Universidade De SÃo PaUlo \\ PARA \\ OBTENÇÃO DO TÍTULO \\ $\mathrm{DE}$ \\ Mestre em CiÊnCIAS \\ Programa: Ciência da Computação \\ Orientador: Prof. Dr. Denis Deratani Mauá
}

Durante o desenvolvimento deste trabalho o autor recebeu auxílio financeiro da CAPES

São Paulo, Novembro de 2017 


\section{Multi-Label Classification based on Sum-Product Networks}

Esta versão da dissertação contém as correções e alterações sugeridas pela Comissão Julgadora durante a defesa da versão original do trabalho, realizada em 06/09/2017. Uma cópia da versão original está disponível no

Instituto de Matemática e Estatística da Universidade de São Paulo.

Comissão Julgadora:

- Prof. Dr. Denis Deratani Mauá - IME-USP

- Prof. Dr. Ricardo Cerri - UFSCar

- Prof. Dr. Fabio Augusto Faria - UNIFESP 


\section{Acknowledgment}

I would like to express my sincere gratitude to my advisor Prof. Dr. Denis Deratani Mauá for the continued support of my research, for his patience, motivation and enthusiasm. The door to Prof. Denis office was always open whenever I had a difficulty or question about my research.

Many thanks to my colleagues and friends Débora, Erika, Paula and Viviane and all others from the LIAMF, for having a nice time and providing an inspiring and scientific atmosphere. I would also thank my Peruvian friends for their help and support.

Finally, I must express my very profound gratitude to God, my family, Ana, Daniel, Ytsmenia, Esmeralda, Antonella and Wilmer, and to my boyfriend, Marcos, for providing me with unfailing support, love and continuous encouragement throughout my master's research. This accomplishment would not have been possible without them. Thank you. 


\section{Resumo}

Villanueva, J. G. Classificação Multi-Rótulo baseada em Redes Soma-Produto . 2017. 43 f. Dissertação Mestrado - Instituto de Matemática e Estatística, Universidade de São Paulo, São Paulo, 2017.

A classificação Multi-Rótulo consiste em aprender uma função que seja capaz de mapear um objeto para um conjunto de rótulos relevantes. Ela possui aplicações como associação de genes com funções biológicas, classificação semântica de cenas e categorização de texto. A classificação tradicional, de rótulo único é, portanto, um caso particular da Classificação Multi-Rótulo, onde cada objeto está associado com exatamente um rótulo.

Uma abordagem bem sucedida para classificação é obter um modelo probabilístico da relação entre atributos do objeto e rótulos. Esse modelo pode então ser usado para classificar objetos, encontrando a predição mais provável por meio da probabilidade marginal ou a explicação mais provavél dos rótulos dados os atributos. Dependendo da família de modelos probabilísticos escolhidos, tais inferências podem ser intratáveis quando o número de rótulos é grande.

As redes Soma-Produto (SPN, do inglês Sum Product Network) são modelos probabilísticos profundos, que permitem inferência marginal tratável. No entanto, como em muitos outros modelos probabilísticos, a inferência da explicação mais provavél é NP-difícil. Embora SPNs já tenham sido usadas com sucesso para tarefas de classificação tradicionais, não existe investigação aprofundada no uso de SPNs para classificação Multi-Rótulo.

Neste trabalho, investigamos o uso de SPNs para classificação Multi-Rótulo. Comparamos vários algoritmos de aprendizado de SPNs combinados com diferentes abordagens propostos para classificação. Mostramos que os classificadores Multi-Rótulos baseados em SPN são competitivos contra classificadores estado-da-arte, como Random k-Labelsets usando Máquinas de Suporte Vetorial e inferência exata da explicação mais provavél em CutNets, em uma coleção de conjuntos de dados de referência.

Plavras-chave: Classificação Multi-Rótulo, modelos probabilísticos, redes suma-produto. 


\section{Abstract}

Villanueva,, J. G. Multi-Label Classification based on Sum-Product Networks. 2017. 43 f. Dissertação Mestrado - Instituto de Matemática e Estatística, Universidade de São Paulo, São Paulo, 2017.

Multi-label classification consists of learning a function that is capable of mapping an object to a set of relevant labels. It has applications such as the association of genes with biological functions, semantic classification of scenes and text categorization. Traditional classification (i.e., single-label) is therefore a particular case of multi-label classification in which each object is associated with exactly one label.

A successful approach to constructing classifiers is to obtain a probabilistic model of the relation between object attributes and labels. This model can then be used to classify objects, finding the most likely prediction by computing the marginal probability or the most probable explanation (MPE) of the labels given the attributes. Depending on the probabilistic models family chosen, such inferences may be intractable when the number of labels is large.

Sum-Product Networks (SPN) are deep probabilistic models, that allow tractable marginal inference. Nevertheless, as with many other probabilistic models, performing MPE inference is NPhard. Although, SPNs have already been used successfully for traditional classification tasks (i.e. single-label), there is no in-depth investigation on the use of SPNs for Multi-Label classification.

In this work we investigate the use of SPNs for Multi-Label classification. We compare several algorithms for learning SPNs combined with different proposed approaches for classification. We show that SPN-based multi-label classifiers are competitive against state-of-the-art classifiers, such as Random k-Labelsets with Support Vector Machine and MPE inference on CutNets, in a collection of benchmark datasets.

Keywords: Multi-label classification, probabilistic graphical models, sum-product networks. 


\section{Contents}

$\begin{array}{ll}\text { List of Abbreviations } & \text { ix }\end{array}$

List of Symbols $\quad$ xi

List of Figures $\quad$ xiii

List of Tables $\quad$ Xv

1 Introduction $\quad 1$

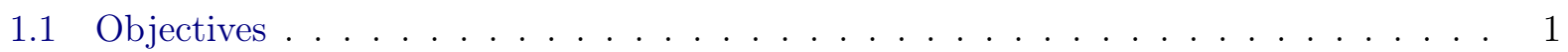

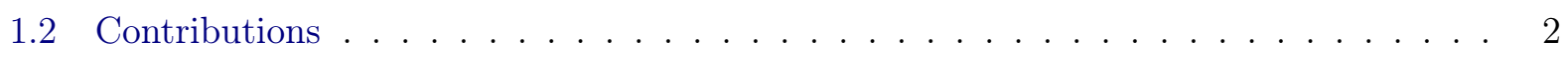

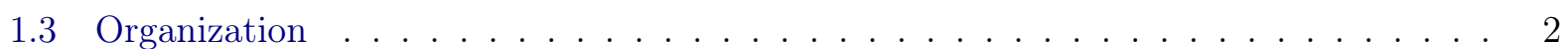

2 Sum-Product Networks $\quad 3$

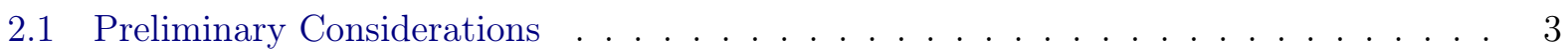

2.2 Sum-Product Networks . . . . . . . . . . . . . . . . . . . . . . . . . . . . . 3

2.2.1 Sum-Product Networks Properties . . . . . . . . . . . . . . . . . . 4

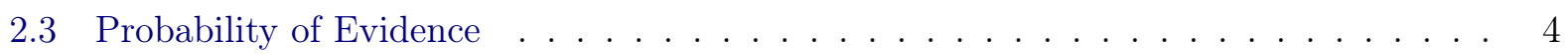

2.4 Most Probable Explanation . . . . . . . . . . . . . . . . . . . . . . 5

2.5 Sum-Product Networks Structure Learning . . . . . . . . . . . . . . . . . . . . 6

2.5.1 LearnSPN . . . . . . . . . . . . . . . . . . . . . . 6

2.5.2 Deepening by limiting node splits $(\mathrm{LL}) \ldots \ldots \ldots \ldots \ldots$

2.5.3 Regularization by tractable multivariate distribution hybridization (LC) $\ldots 9$

2.5.4 Learning with Direct and Indirect Variable Interactions (ID) . . . . . . . . . 10

3 Multi-Label Classification $\quad 15$

3.1 Methods for Multi-Label Classification . . . . . . . . . . . . . . . . . 16

3.1 .1 Binary Relevance . . . . . . . . . . . . . . . . . . 16

3.1 .2 Chain Classifier . . . . . . . . . . . . . . . . . . . 16

3.1 .3 Label Power-Set . . . . . . . . . . . . . . . . . . . . . . . 17

3.1.4 Probabilistic Graphical Models . . . . . . . . . . . . . . . . . . . 17

4 Multi-Label Classification based on Sum-Product Networks $\quad 19$

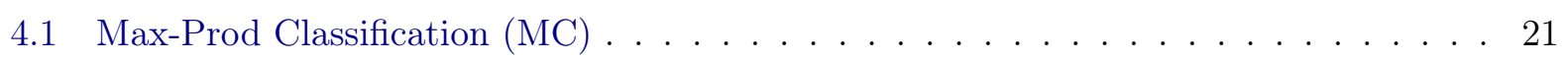

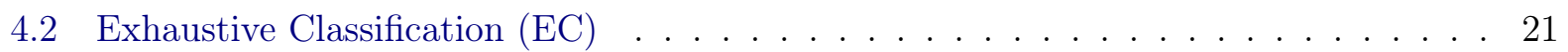

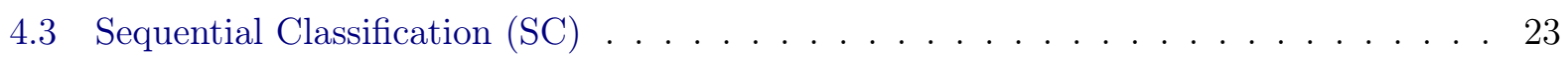




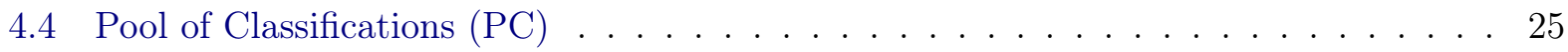

4.5 Complexity Analysis . . . . . . . . . . . . . . . . . . . . . 27

5 Experiments $\quad 29$

5.1 Datasets . . . . . . . . . . . . . . . . . . . . . . . . . 29

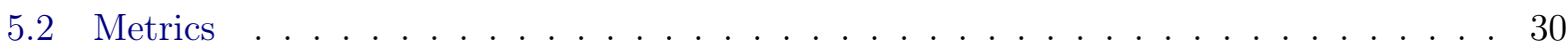

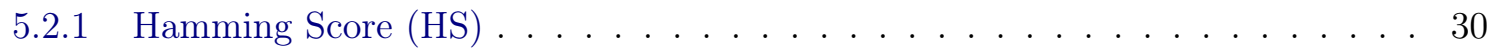

5.2 .2 Exact Match $(\mathrm{EM}) \ldots \ldots \ldots \ldots \ldots \ldots$

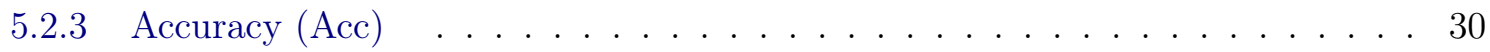

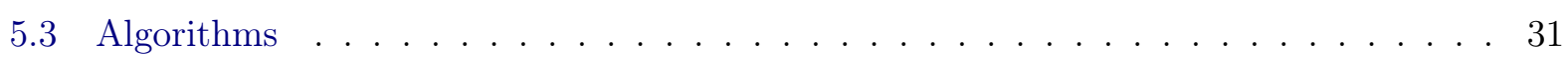

5.3.1 Multi-label Classifiers based on $\mathrm{SPN} \ldots \ldots \ldots \ldots$. . . . . . . . . 31

5.3 .2 State-Of-Art Multi-Label Classifiers . . . . . . . . . . . . . . . . 32

5.4 Results and Discussion for Multi-Label Classifiers based on SPN and State-of-art . . 32

5.4.1 Comparing classifiers with Wilcoxon signed-rank test . . . . . . . . . . 34

5.4.2 Comparing classifiers with Bayesian signed-rank test . . . . . . . . . . . . . 34

6 Conclusions $\quad 39$

6.1 Suggestions for Future Research . . . . . . . . . . . . . . . . . . . 39

$\begin{array}{ll}\text { Bibliography } & 41\end{array}$ 


\title{
List of Abbreviations
}

\author{
MLC Multi-Label Classification \\ MPE Most Probable Explanation \\ SPN Sum-Product Network \\ SVM Support Vector Machine \\ RCNET Multi-label classification on CutNets with MPE exact computation \\ RSVM Multi-label classification Random k-labelset using SVM \\ RV Random Variable \\ MCC Multi-Class Classification \\ BC Binary Classification \\ LL Learning SPN algorithm limiting node splits \\ LC Learning SPN algorithm with Chow-Liu Trees as leaves \\ ID Learning SPN algorithm with direct and indirect variable interactions \\ BR Binary Relevance approach for MLC \\ CC Classifier Chain approach for MLC \\ LP Label Power-set approach for MLC \\ PGM Probabilistic Graphical Models \\ EC Exhaustive Classification approximate MPE algorithm \\ MC Max-Prod Classification approximate MPE algorithm \\ SC Sequential Classification approximate MPE algorithm \\ PSC Pool of Sequential Classification approximate MPE algorithm \\ PEC Pool of Exhaustive Classification approximate MPE algorithm \\ Acc Accuracy metric for Multi-Label Classification \\ HS Hamming Score metric for Multi-Label Classification \\ EM Exact Match metric for Multi-Label Classification \\ EC+LL Multi-Label Classifier based on SPNs using EC and LL algorithms \\ EC + LC Multi-Label Classifier based on SPNs using EC and LC algorithms \\ EC+ID Multi-Label Classifier based on SPNs using EC and ID algorithms \\ $\mathrm{MC}+\mathrm{LL}$ Multi-Label Classifier based on SPNs using MC and LL algorithms \\ $\mathrm{MC}+\mathrm{LC}$ Multi-Label Classifier based on SPNs using MC and LC algorithms \\ $\mathrm{MC}+\mathrm{ID}$ Multi-Label Classifier based on SPNs using MC and ID algorithms
}


SC+LL Multi-Label Classifier based on SPNs using SC and LL algorithms

$\mathrm{SC}+\mathrm{LC} \quad$ Multi-Label Classifier based on SPNs using SC and LC algorithms

SC+ID Multi-Label Classifier based on SPNs using SC and ID algorithms

PSC + LL Multi-Label Classifier based on SPNs using PSC and LL algorithms

PSC+LC Multi-Label Classifier based on SPNs using PSC and LC algorithms

PSC + ID Multi-Label Classifier based on SPNs using PSC and ID algorithms

PEC + LL Multi-Label Classifier based on SPNs using PEC and LL algorithms

PEC + LC Multi-Label Classifier based on SPNs using PEC and LC algorithms

PEC+ID Multi-Label Classifier based on SPNs using PEC and ID algorithms 


\section{List of Symbols}

$\begin{array}{ll}\mathcal{S} & \text { Sum-Product Network } \\ \mathcal{P}_{\mathcal{S}} & \text { SPN distribution } \\ \mathcal{S}_{i} & \text { Sub-SPN rooted at node } i \\ \mathcal{S}^{m} & \text { Max-Product Network } \\ V & \text { Random Variable } \\ \mathbf{V} & \text { Set of Random variables } \\ \operatorname{val}(V) & \text { Set of values of } V \\ v & \text { A value of } V \text {, where } v \in \operatorname{val}(V) \\ \mathcal{Z} & \text { Partition function } \\ \alpha & \text { Parameter for Laplace smooth } \\ \mathcal{M} & \text { A Markov Network } \\ \mathcal{P}_{\mathcal{M}} & \text { Markov Network distribution } \\ \emptyset & \text { Empty set } \\ \Delta_{l l} & \text { Increase of log-likelihood } \\ \Delta_{e} & \text { Increase of edges } \\ \gamma & \text { User defined weight } \\ \mathcal{C} & \text { Classifier } \\ \mathcal{D} & \text { Training set } \\ \mathcal{D}_{j} & \text { Training subset } \\ \theta & \text { Labels ordering } \\ \mathcal{C S} & \text { Set of classifications } \\ & \end{array}$




\section{List of Figures}

2.1 Sum-Product Networks examples . . . . . . . . . . . . . . . . . . . . . 3

2.2 Sum-Product Networks properties . . . . . . . . . . . . . . . . . . 4

2.3 Example of computation evidence probability on $\mathrm{SPN} \ldots \ldots \ldots$

2.4 Example of Max-Product algorithm on SPNs . . . . . . . . . . . . 6

2.5 Algorithm LearnSPN . . . . . . . . . . . . . . . . . . . . . 7

2.6 Algorithm LearnSPN limiting on node splits . . . . . . . . . . . . . . . . . 8

2.7 Algorithm LearnSPN with multivariate distribution hybridization . . . . . . . . . . 10

2.8 Arithmetic Circuit . . . . . . . . . . . . . . . . . . . 11

2.9 Example of SPN learned with ID algorithm . . . . . . . . . . . . . . . 12

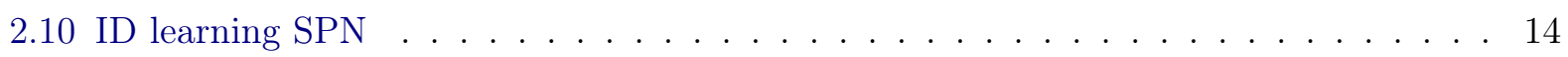

3.1 Multi-Label Dataset representation . . . . . . . . . . . . . . . . . 15

3.2 Examples of Traditional, Multi-Class and Multi-Label classifications . . . . . . . . 15

3.3 Example of Binary Relevance training dataset transformation . . . . . . . . . . . 16

3.4 Example of Chain Classifier training dataset transformation . . . . . . . . . . . . . 16

3.5 Label Power-Set training dataset Transformation . . . . . . . . . . . . . . . . 17

3.6 An example of a probabilistic model . . . . . . . . . . . . . . . . . 18

4.1 Multi-label classifier based on SPN adopting BR approach . . . . . . . . . . . . . 19

4.2 Multi-label classifier based on SPN adopting CC approach . . . . . . . . . . . . . 20

4.3 Probability distributions of variable $\mathrm{A}$ and $\mathrm{B} \ldots \ldots \ldots \ldots$

4.4 Multi-label classifier based on SPN using Max-Prod classification . . . . . . . . . . 21

4.5 An example of EC optimization . . . . . . . . . . . . . . . . . . 22

4.6 An example of Exhaustive classification . . . . . . . . . . . . . . . . . . . . . . . . . . . . . . . . . . .

4.7 An example of sequential classification . . . . . . . . . . . . . . . . . . . 24

4.8 An example of pool classification . . . . . . . . . . . . . . . . . 25

4.9 An example of Pool Sequential Classification _ . . . . . . . . . . . . . . . 26

4.10 An example of Pool Exhaustive Classification . . . . . . . . . . . . . . 27

5.1 Pairwise Bayesian signed-rank test for Accuracy metric . . . . . . . . . . . . 35

5.2 Pairwise Bayesian signed-rank test for Exact Match metric . . . . . . . . . . . 36

5.3 Pairwise Bayesian signed-rank test for Hamming Score metric . . . . . . . . . . . . 37

5.4 Posterior graphic from the Bayesian signed-rank test for Accuracy, Exact Match and Hamming Score . . . . . . . . . . . . . . . . . . . . . . 38 


\section{List of Tables}

5.1 Multi-Label Datasets . . . . . . . . . . . . . . . . . . . . . . . . 29

5.2 Multi-Label Classifiers based on Sum-Product Networks . . . . . . . . . . . . . . 31

5.3 Comparison Hamming Score ． . . . . . . . . . . . . . . . . . . 32

5.4 Comparison Exact Match . . . . . . . . . . . . . . . . 33

5.5 Comparison Accuracy . . . . . . . . . . . . . . . . . . . . . 33

5.6 P-value obtained from the Wilcoxon signed-rank test on the Compared classifiers for each Metric . . . . . . . . . . . . . . . . . . . . . . . 34 


\section{Chapter 1}

\section{Introduction}

Multi-Label Classification (MLC) consists in learning a function that associates each example, represented by a feature vector, to a set of (relevant) labels. It therefore generalizes traditional (single-label) classification problem, where each example is associated with a single label. Categorizing music into emotions [TTKV08], associating genes to biological functions [CK01] and performing semantic classification of scenes [BLSB04] are some examples of MLC applications.

A popular and often effective approach to MLC is to transform the problem into one or many single-label classification problems. For example, the Binary Relevance approach consists in independently learning a binary classifier for each distinct label using only the feature vectors [TK06]. Even though it ignores label correlations, this approach often performs reasonably.

A different approach that tries to capture label correlation is the Classifier Chain [DWCH12]. This approach takes an ordering of the labels and sequentially learns a single-label classifier using (in addition to the original attributes) the smaller labels as attributes [RPHF11]. Selecting an ordering of the labels is not trivial and might greatly affect the classification performance.

A caveat of both these approaches is that they require learning a number of classifiers that is equal to the number of labels; since learning a classifier is usually expensive, this might scale poorly to problems with hundreds or even thousands of labels, as is the case in many real domains.

A more principled approach to capture label correlations while dispensing with the need of learning a large number of models is to learn a single joint probabilistic model, such as a Bayesian Network or a Markov Network, over the features and label variables [CAMG14, ACMG13, FGG97, LMP01]. The probabilistic model can then be used to classify the labels either by maximizing the marginal probability of each label given the features, which can result in inconsistent predictions; or by computing the Most Probable Explanation (MPE) of the labels given the features, that maximizes the join probability of the classification given the features.

Sum-product networks (SPNs) are a relatively new class of deep probabilistic models that have obtained impressive results in many tasks such as image completion [PD11] and image (singlelabel) classification [GD12, SC16, ABG15]. An SPN represents a probability distribution over a set of random variables by a rooted directed-acyclic graph with tractable distributions as leaves, sums and products as inner nodes and weighted edges. Although SPNs have already been show to perform well in specific MLC tasks (e.g., image completion), there has been no in-depth investigation of the use of SPNs to tackle general MLC problems.

\section{$1.1 \quad$ Objectives}

This work aims to investigate the adequacy of SPNs for MLC. Our approach learns a single SPN from data and then uses it to obtain the classification given features as evidence. We experiment with several different state-of-the-art structure learning algorithms for learning SPNs.

An approach for classification using a SPN is to maximize the marginal probability for each label given the features as evidence, this approach is often effective since SPNs computes marginal inference in linear time in its size. However it can result in low probability label sets. 
Another approach consists in compute the MPE for the labels, finding the most probable configuration of relevant labels given the features, as a consequence obtains predictions with high joint probability. Unfortunately compute exact MPE on SPNs is NP-hard [PGPD16].

Aiming to construct efficient classifiers based on SPNs, which map objects to consistent predictions in a reasonable time, we propose several new classification algorithms, and compare them against each other, the widely-used max-product algorithm [PD11], which approximates the MPE by replacing sum by maximizations in the evaluation of the SPN, the state-of-the-art Multi-Label classifiers RSVM and RCNET.

\subsection{Contributions}

The main contributions of this work are the proposed algorithms for Multi-Label classification using a single Sum-Product Network, which are described below:

\section{- Exhaustive Classification}

Search exhaustively for the most likely prediction in a set of candidates, these candidates are obtained by choosing all distinct classifications on the training set, and then selects the relevant labels that maximize the probability of each candidate given the features.

\section{- Sequential Classification}

In the spirit of the Classifier Chain, takes a linear ordering over the labels and sequentially selects the maximum probability value for the current label given the smaller labels and features. We apply two criteria for choosing that label ordering: Static Best-First, orders label by the marginal probability of relevance given features only. Dynamic Best-First selects at each iteration the label with the current highest probability of relevance given features and previously selected labels.

\section{- Pooling classifications}

Based on ensemble of classifications, obtains a set of classification from Sequential Classification using random orderings or from the set of most probable predictions by Exhaustive Classification. Combines these classifications by computing the weighted average of each marginal probability (given features and previous labels), computing the majority voting for each label, and simply selecting the classification with higher probability.

Our results show that our proposed SPN-based Multi-label classifiers, obtained by different combinations of learning and classification algorithms, in average for Accuracy, Hamming Score and Exact Match metrics, outperforms the state-of-the-art Multi-Label classifiers RSVM, that adopts the Random k-labelset approach with Support Vector Machines as basis multi-class classifier and RCNET, that compute exact MPE on CutsetNets in a collection of benchmark datasets.

Part of the contributions of this work was published in the 6th Brazilian Conference on Intelligent Systems (BRACIS) [LM17].

\subsection{Organization}

The remainder of the paper is organized as follows. In Chapter 2, we introduce some basic knowledge about SPNs and discuss state-of-the-art methods for structure learning. In Chapter 3, we introduce basic concepts about Multi-Label Classification. In Chapter 4 we present our approach for MLC based on SPNs. In Chapter 5 we describe the experiments and show the experimental results of the proposed classifiers. Finally, we conclude the work and discuss possible improvements in Chapter 6. 


\section{Chapter 2}

\section{Sum-Product Networks}

\subsection{Preliminary Considerations}

We begin by establishing some notation. Random variables (RV) are denoted by upper-case letters $(V)$. The set of values of an RV $V$ is denoted by $\operatorname{val}(V)$, where $v \in \operatorname{val}(V)$. Sets of RVs are written in bold $(\mathbf{V})$. For RVs set $\mathbf{V}=\left\{V_{1}, V_{2}, \ldots, V_{N}\right\}$, we define $\operatorname{val}(\mathbf{V})=\times_{n=1}^{N} \operatorname{val}\left(V_{n}\right)$, where $\mathbf{v} \in$ $\operatorname{val}(\mathbf{V})$.

\subsection{Sum-Product Networks}

An SPN is a rooted directed acyclic graph with tractable distributions as leaves, sums and products as internal nodes, and non-negative weights associated to the edges connecting sum nodes to their children [PD11]. An SPN $\mathcal{S}$ represents a tractable non-normalized probability distribution $\mathcal{P}_{\mathcal{S}}$ over a set of RVs $\mathbf{V}$. A distribution is called tractable if any marginal probability induced by it can be computed in linear time.

Figure 2.1 contains examples of valid SPNs, that is SPNs representing valid distributions, Figure 2.1a shows an SPN with univariate distributions as leaves, while Figure 2.1b shows a SPN with multivariate distributions (Chow-Liu Trees ${ }^{1}$ [CL68]) as leaves.

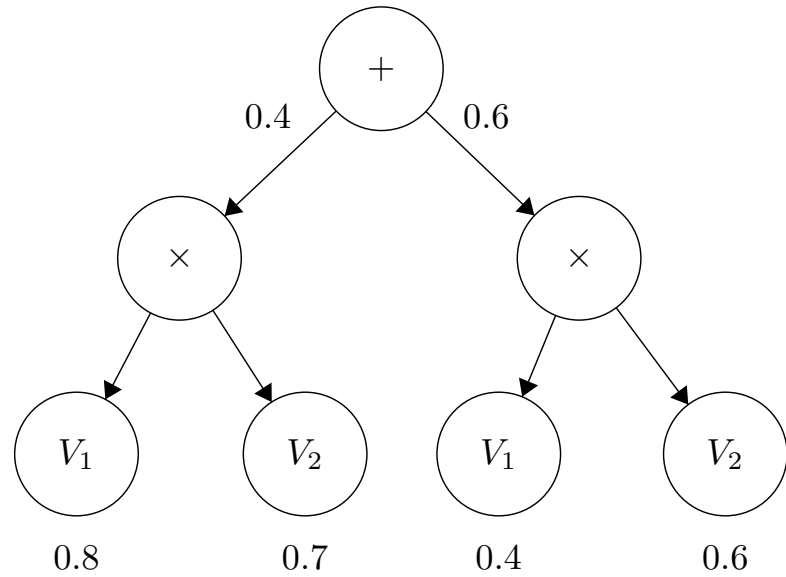

(a)

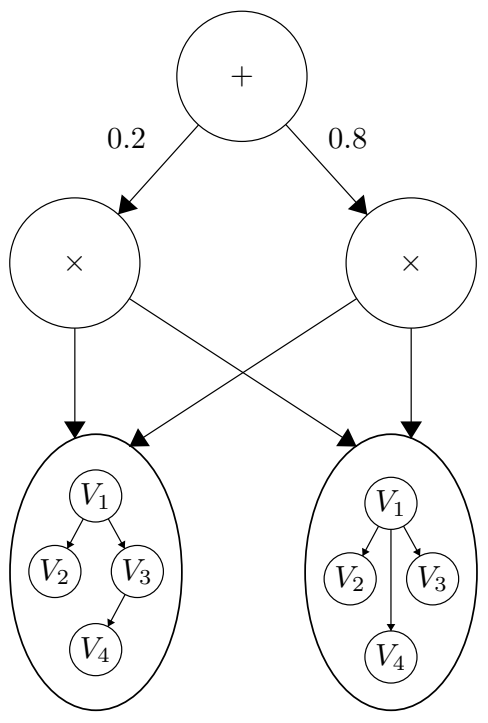

(b)

Figure 2.1: SPNs with univariate distributions (2.1a) and Chow-Liu trees (2.1b) as leaves. The numbers below the leaves in (2.1a) denote the probability that the corresponding variables takes on value 1.

\footnotetext{
${ }^{1}$ Chow-Liu Trees are Bayesian Networks where each node have at most one parent
} 


\subsubsection{Sum-Product Networks Properties}

The scope of an SPN, is the set of RVs appearing in their leaves distribution. The scope of a node is the scope of the corresponding Sub-SPN rooted at that node. The set of all nodes of an SPN satisfies the following properties to ensure that it represents a valid distribution:

- The children of a sum node have identical scope (Completeness).

- The children of a product node have pairwise disjoint scopes (Decomposability).

The Figure 2.2 shows that the Sum-Product Network at the Figure 2.1a represent a valid distribution and satisfies completeness and decomposability properties.

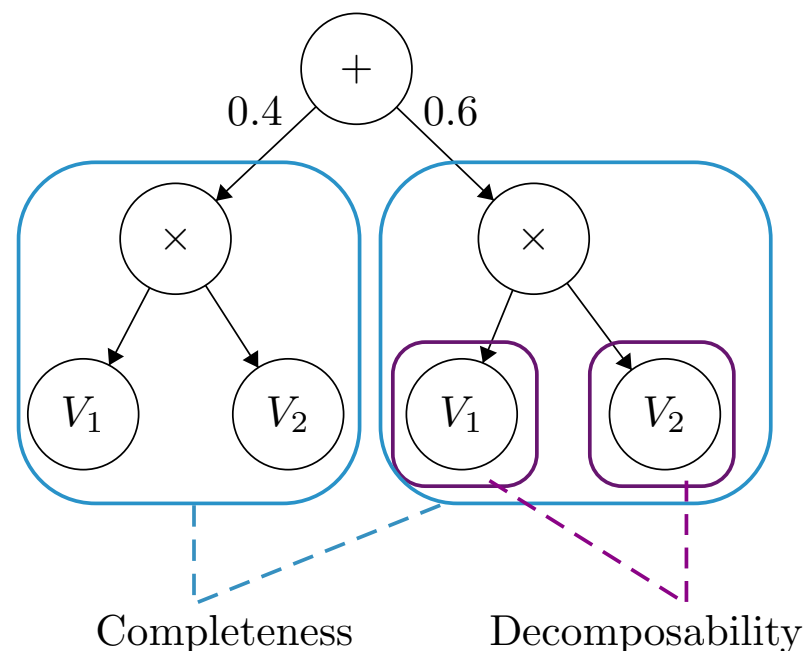

Figure 2.2: Completeness and Decomposability properties in the SPN of the Figure 2.1a

\subsection{Probability of Evidence}

For the SPN $\mathcal{S}$ with scope $\mathbf{V}$, we write $\mathcal{S}(\mathbf{e})$ to denote the network evaluation at $\mathbf{e}$, where $\mathbf{E} \subseteq \mathbf{V}$. An SPN defines a (normalized) probability distribution by:

$$
\mathcal{P}_{\mathcal{S}}(\mathbf{E}=\mathbf{e})=\frac{\mathcal{S}(\mathbf{e})}{\mathcal{Z}}
$$

where $\mathcal{Z}=\sum_{\mathbf{v}} \mathcal{S}(\mathbf{v})$ is the partition function of the network. If the weights at each sum node in $\mathcal{S}$ add to one and the distributions at the leaves are normalized, then $\mathcal{Z}=1$ and $\mathcal{S}$ defines a probability distribution by:

$$
\mathcal{P}_{\mathcal{S}}(\mathbf{E}=\mathbf{e})=\mathcal{S}(\mathbf{e})
$$

The upward evaluation of the network $\mathcal{S}$ at $\mathbf{e}$ is computed as follows:

- First evaluates the leaves values. Let $\mathbf{A}$ be the set of variables in the leaf $i$ :

- If $\mathbf{A} \subseteq \mathbf{E}$, the value of $i$ in $\mathcal{S}$ is simply the value $\mathcal{S}_{i}(\mathbf{e})=\mathcal{P}_{i}(\mathbf{A}=\mathbf{a})$ of the corresponding probability distribution $\mathcal{P}_{i}$.

- Otherwise the value of $i$ in $\mathcal{S}$ is one, $\mathcal{S}_{i}(\mathbf{e})=1$, summing over the variables in $\mathbf{A}$.

- The value of a sum node $i$ is $\mathcal{S}_{i}(\mathbf{e})=\sum_{i \rightarrow j \in \mathcal{S}} w_{i j} \mathcal{S}_{j}(\mathbf{e})$, where the sum ranges over the children of $i$ and $w_{i j}$ is the weight associated with the edge $i \rightarrow j$.

- The value of a product node $i$ is $\mathcal{S}_{i}(\mathbf{e})=\prod_{i \rightarrow j \in \mathcal{S}} \mathcal{S}_{j}(\mathbf{e})$, where $j$ and $i \rightarrow j$ are as before. 
- Finally, the SPN value at $\mathbf{e}, \mathcal{S}(\mathbf{e})$, is the value of its root.

For example, in the SPN in Figure 2.1a:

- For $\mathbf{e}=\left\{V_{1}=1, V_{2}=0\right\}, \mathcal{S}(\mathbf{e})=0.4(0.8 \times 0.3)+0.6(0.4 \times 0.4)=0.192$.

- For $\mathbf{e}=\left\{V_{1}=0\right\}, \mathcal{S}(\mathbf{e})=0.4(0.2 \times 1)+0.6(0.6 \times 1)=0.44$. Figure 2.3 shows the computation of probability on the network.

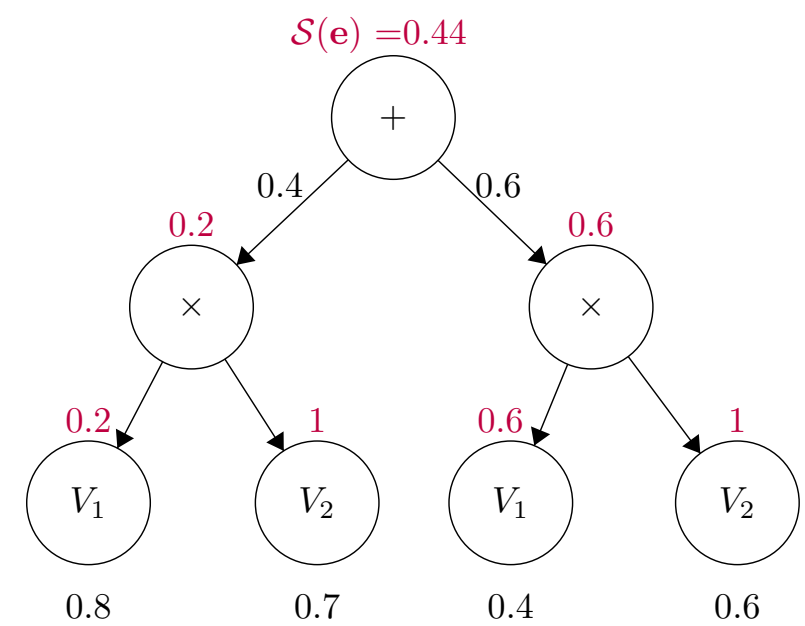

Figure 2.3: Computation of the probability of evidence on the $S P N$ in the Figure 2.1a and $\boldsymbol{e}=\left\{V_{1}=0\right\}$, where the node value is in purple above of the corresponding node. The resulting probability is $\mathcal{P}_{\mathcal{S}}(\boldsymbol{e})=0.44$

\subsection{Most Probable Explanation}

The problem of finding a most probable configuration given evidence has many applications such as Multi-Label Classification, that aims to find the most probable configuration of relevant labels given the object features.

Since an SPN defines a distribution, we can obtain the Most Probable Explanation (MPE) of a network $\mathcal{S}$ by:

$$
\underset{\mathbf{q}}{\operatorname{argmax}} \mathcal{P}_{\mathcal{S}}(\mathbf{q} \mid \mathbf{e})=\underset{\mathbf{q}}{\operatorname{argmax}} \mathcal{P}_{\mathcal{S}}(\mathbf{q}, \mathbf{e})
$$

where $\mathbf{e}$ is a configuration of the observed variables $\mathbf{E}, \mathbf{Q}$ is a set of query variables and $\mathbf{E}, \mathbf{Q} \subset \mathbf{V}$. Unfortunately, to find a MPE on Sum-Product Networks is NP-hard [PGPD16], even when restricted to binary RVs.

A popular approximate algorithm proposed by Poon and Domingos consists in obtaining a MaxProduct Network (MPN) $\mathcal{S}^{m}$ by replacing the sums nodes in an SPN $\mathcal{S}$ with maximization nodes [PD11], and traverse the network $\mathcal{S}^{m}$ twice:

- The first pass computes a lower bound on the probability of the MPE by evaluating the network bottom-up as usual, but labeling maximizers at each max node.

- The second pass finds the corresponding configuration by starting at the root and then recursively selecting a highest-valued child of a max node, all children of a product node and an MPE of each leaf.

For example, for the SPN in Figure 2.1a to find the approximate MPE of $V_{1}$ given $V_{2}=$ $1, \operatorname{argmax}_{v_{1}} \mathcal{P}_{\mathcal{S}}\left(v_{1} \mid V_{2}=1\right)$, first obtains the MPN from the SPN and execute the first pass: 
$\max (0.4(0.8 \times 0.3), 0.6(0.6 \times 0.4))=0.144$ and finally recovers the correspondent value $V_{1}=0$ in the MPN path on the second pass. The Figure 2.4 shows in more detail this procedure for the given example.

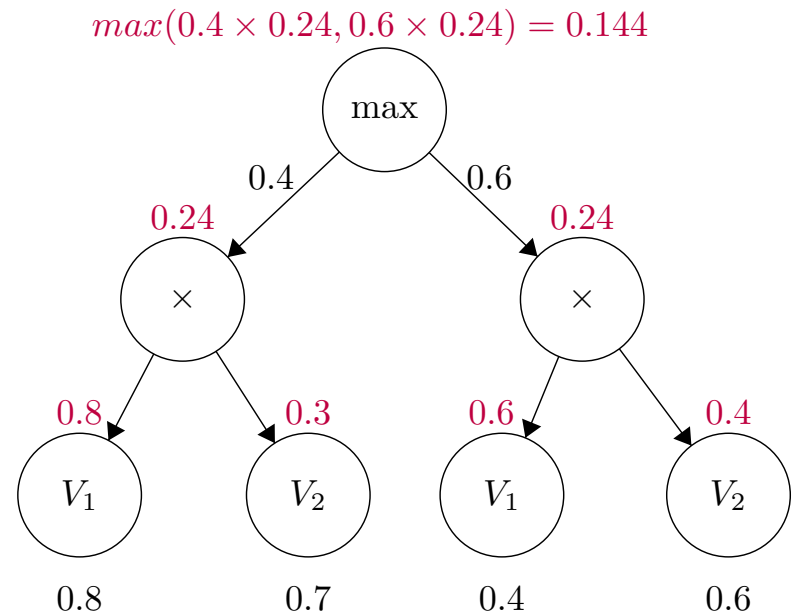

(a)

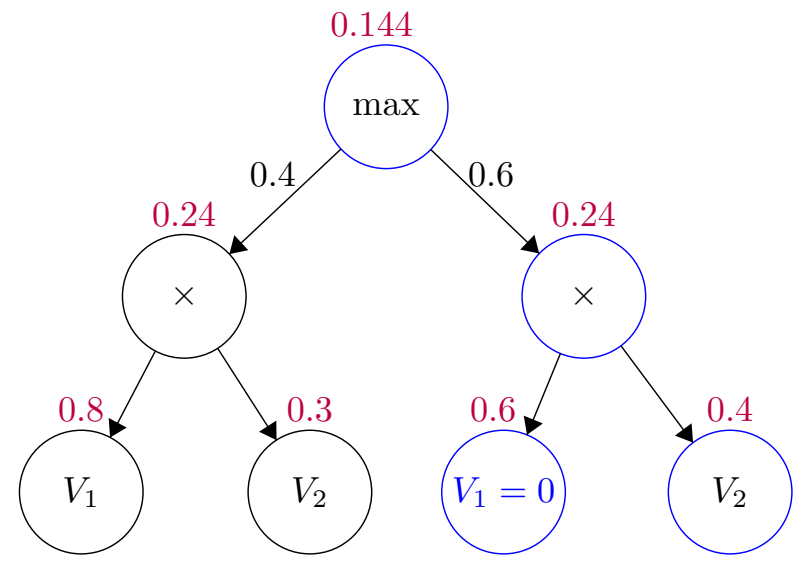

0.8
0.4

0.6

(b)

Figure 2.4: $M P E$ approximation by Max-Prod algorithm on the $S P N$ in Figure 2.1a. For $\boldsymbol{Q}=\left\{V_{1}\right\}$ and $\boldsymbol{e}$ $=\left\{V_{2}=1\right\}$. The Figure 2.4a shows the first pass, obtaining the value of each node (number in purple above of the correspondent node), and the Figure 2.4b shows the second pass on the network recovering the MPE for $\boldsymbol{Q}$ (the selected path on the $M P N$ is in blue).

Unfortunately, computing MPE by the approximate MPN algorithm does not always generate correct results. For the previous example, the MPE calculate by MPN is $V_{1}=0$ given $V_{2}=0$ as evidence, while the exact MPE obtained by computing $\mathcal{P}_{\mathcal{S}}\left(V_{1}=0, V_{2}=0\right)=0.168$ and $\mathcal{P}_{\mathcal{S}}\left(V_{1}=\right.$ $\left.1, V_{2}=0\right)=0.192$ is $V_{1}=1$.

\subsection{Sum-Product Networks Structure Learning}

There are several methods for learning the structure and parameters of an SPN from data [PD11, GD13, DV12, PGP13]. In this work we consider several variants of LearnSPN, that is a simple and popular method to learn both structure and parameters of an SPN [GD13].

\subsubsection{LearnSPN}

LearnSPN is an algorithm schema, that can incorporate a variety of methods for splitting variables and examples into subsets [GD13].

Given a dataset of i.i.d. ${ }^{2}$ examples (configurations) $\mathbf{t}$ of categorical RVs $\mathbf{V}$ (for each $t \in \mathbf{t}, t \in$ $\operatorname{val}(\mathbf{V}))$, LearnSPN operates recursively as follows:

- If $\mathbf{V}$ contains only one variable, return the corresponding univariate distribution (obtained e.g. by maximum likelihood estimation).

- If $\mathbf{V}$ contains more than one variable execute recursive calls for subsets with fewer variables or examples obtained as follows:

- If it is able to split $\mathbf{V}$ into mutually independent subsets (according to the result of some statistical test, such as G-test of pairwise independence), returns a product node whose children are the resulting SPNs obtained from the execution of the recursive calls on each of the mutually independent subsets.

\footnotetext{
${ }^{2}$ Independent and identically distributed RVs
} 
- Otherwise, it performs clustering on the examples $\mathbf{t}$ (according to some clustering algorithm such as Expectation Maximization), creates a sum node whose children are the SPNs retrieved from recurring the procedure in each of the obtained subsets, and whose weights are the proportions of examples in the corresponding subsets.

This method for learning structure and parameters of an SPN is summarized in the Algorithm 1 and illustrated in the Figure 2.5.
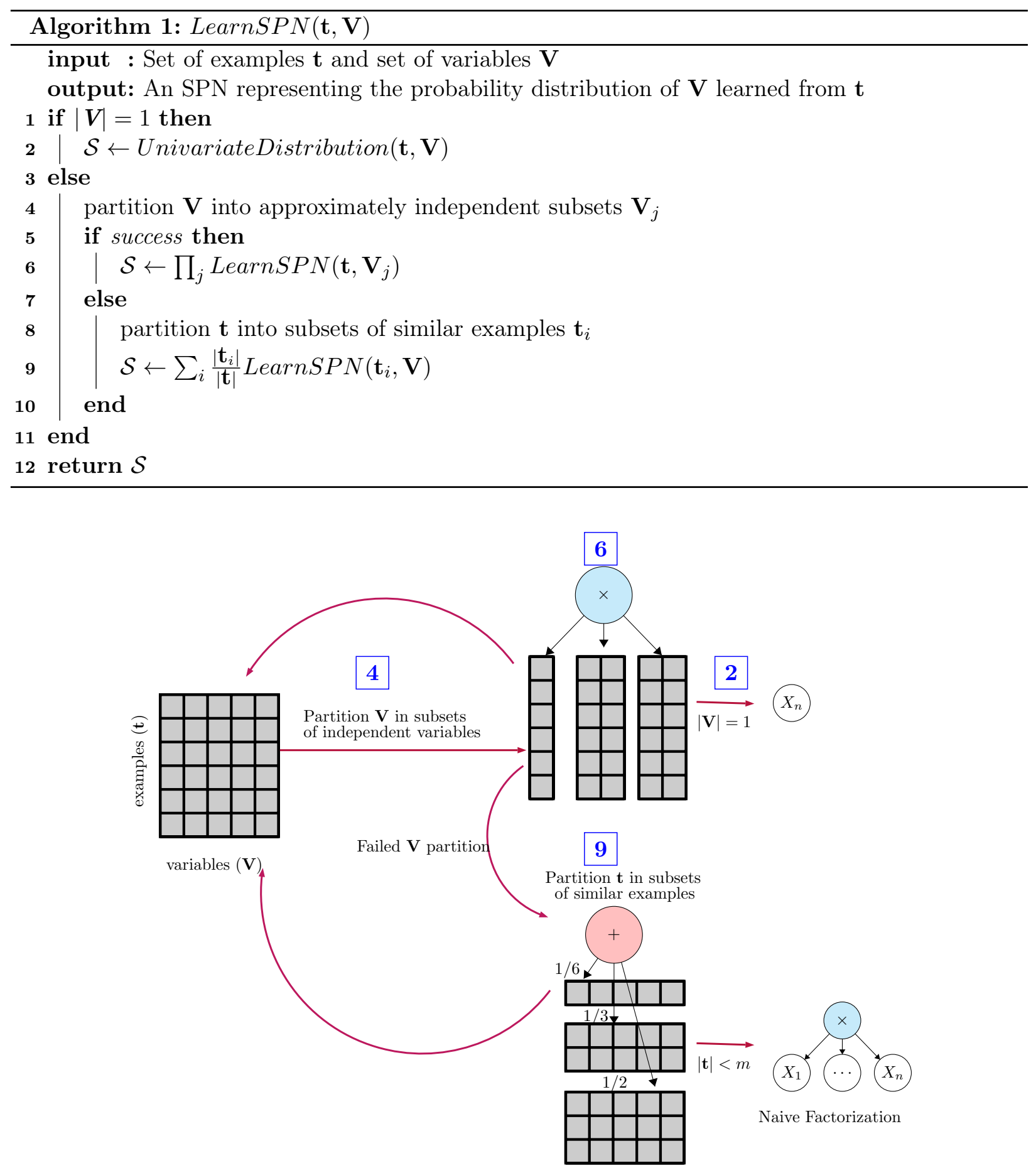

Figure 2.5: Algorithm LearnSPN, that learns the structure and parameters of an SPN from data. The numbers in blue rectangles represent the associate lines number in the Algorithm 1. 


\subsubsection{Deepening by limiting node splits (LL)}

This method improves over LearnSPN by limiting the number of node's children while learning, resulting in deeper networks, and potentially less edges and parameters [VDME15]. The number of subsets in which the current set can be split is usually set to two.

The Algorithm 2 shows the previously described improves over LearnSPN, establishing thresholds for the number of variables partitions $(I)$ and for the examples partitions $(C)$. Additionally limits the minimum number of examples to be evaluated, if an example partition is performed with a number of examples under $m$ creates and returns a product node with univariate distributions as leaves (Naive Factorization). The Figure 2.6 illustrates the LL learning method.

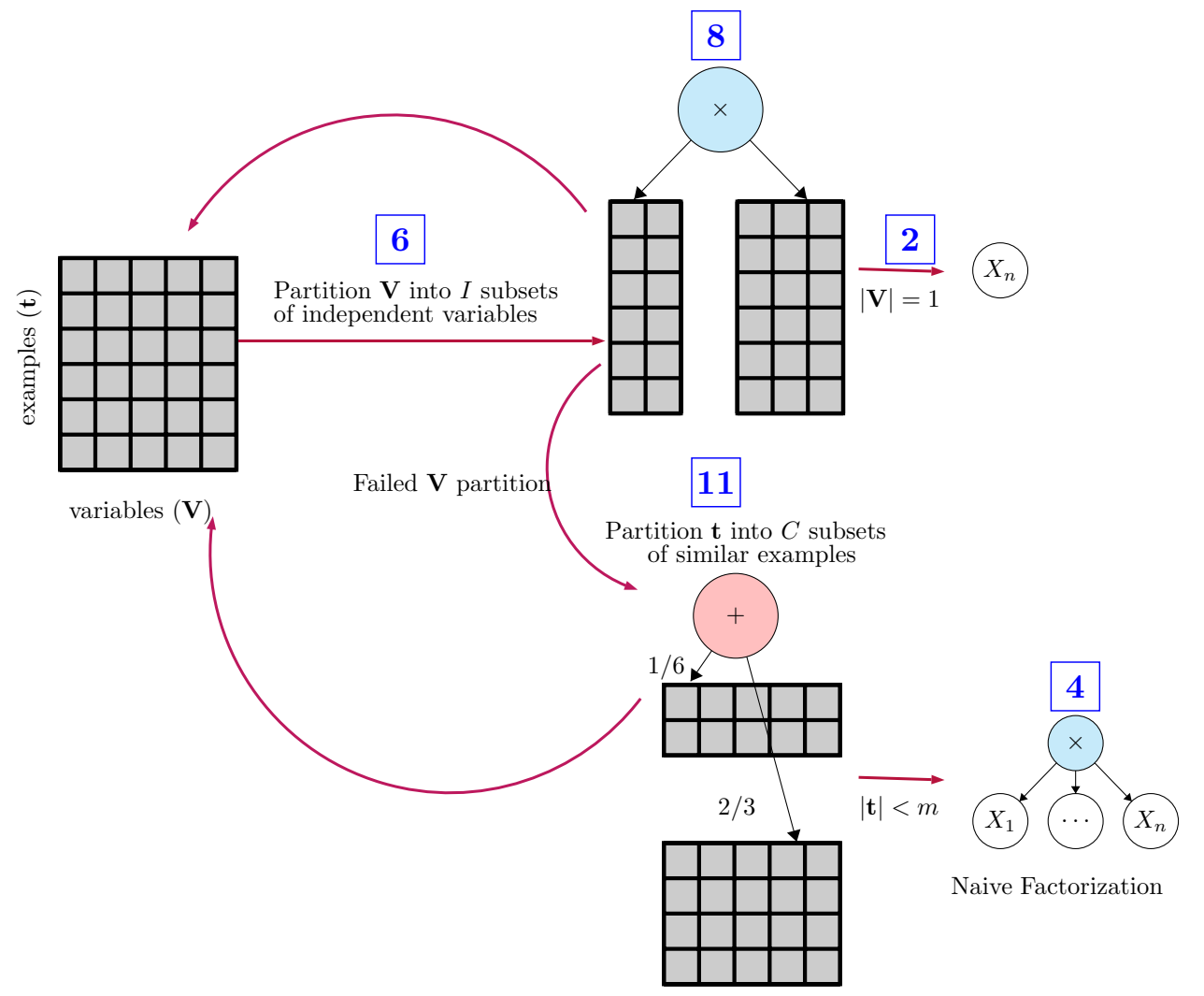

Figure 2.6: Algorithm LearnSPN limiting node splits to two $(I=2$ and $C=2)$. The numbers in blue rectangles represent the associate line number in the Algorithm 2. 

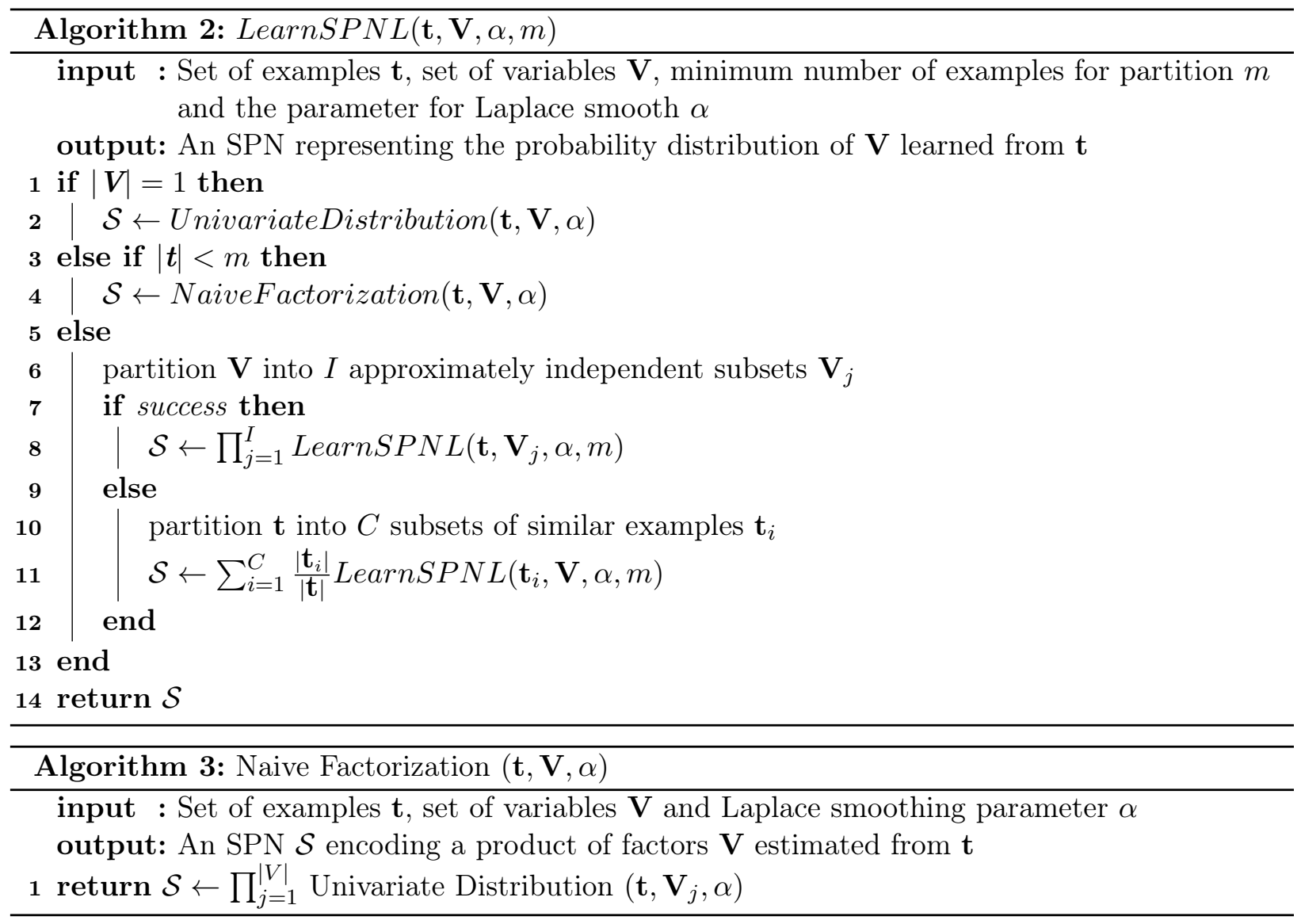

\subsubsection{Regularization by tractable multivariate distribution hybridization (LC)}

Builds on LL, by replacing the naive factorizations by directed tree distributions (Chow-Liu distribution) as the leaves [VDME15]. This increases network data likelihood by enabling the capture of finer local dependencies, while maintaining the overall computational complexity and tractability of distributions (since both marginal inference and MPE can be computed efficiently in directed trees).

For the implementation of the described improvement it is necessary a modification of the Algorithm 2 replacing the line $4 . \mathcal{S} \leftarrow$ NaiveFactorization $(\mathbf{t}, \mathbf{V}, \alpha)$ (creation of naive factorizations) by $4 . \mathcal{S} \leftarrow C L T r e e(\mathbf{t}, \mathbf{V}, \alpha)$ (directed tree distributions learning). CLTree procedure builds the maximum spanning tree on the graph obtained by calculating the mutual information for each pair of variables in $\mathbf{V}$, then transforms the resulting non-directed tree into a directed tree by randomly selecting a root and visiting each node exactly once. Therefore, the leaves of the network are Chow-Liu (multivariate) distributions instead of univariate distributions. The CLTree procedure is summarized in Algorithm 4 and the learning algorithm is illustrated in Figure 2.7.

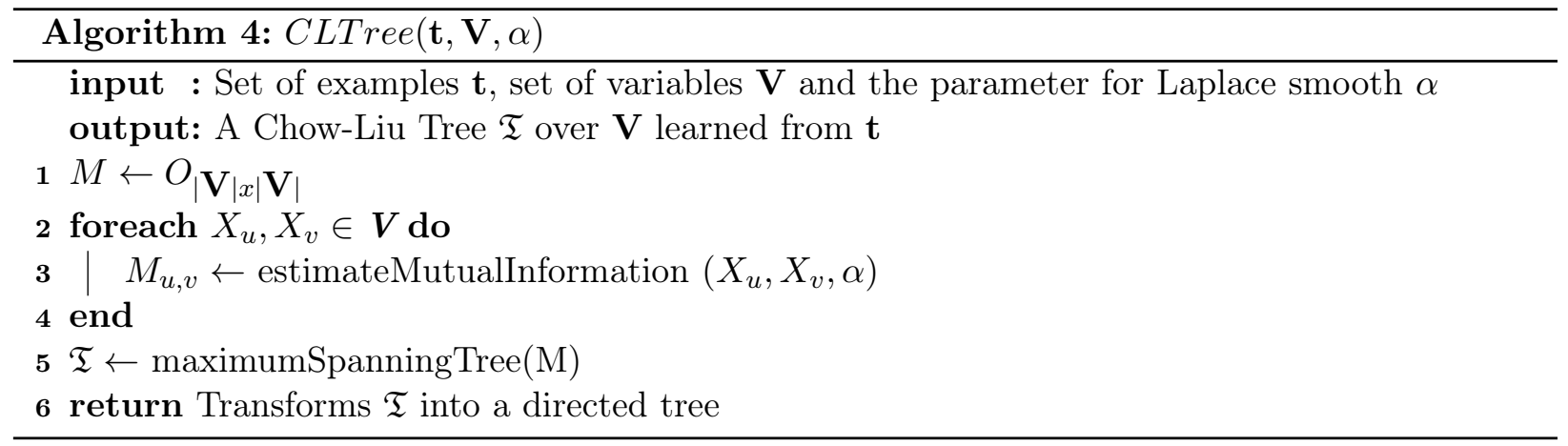




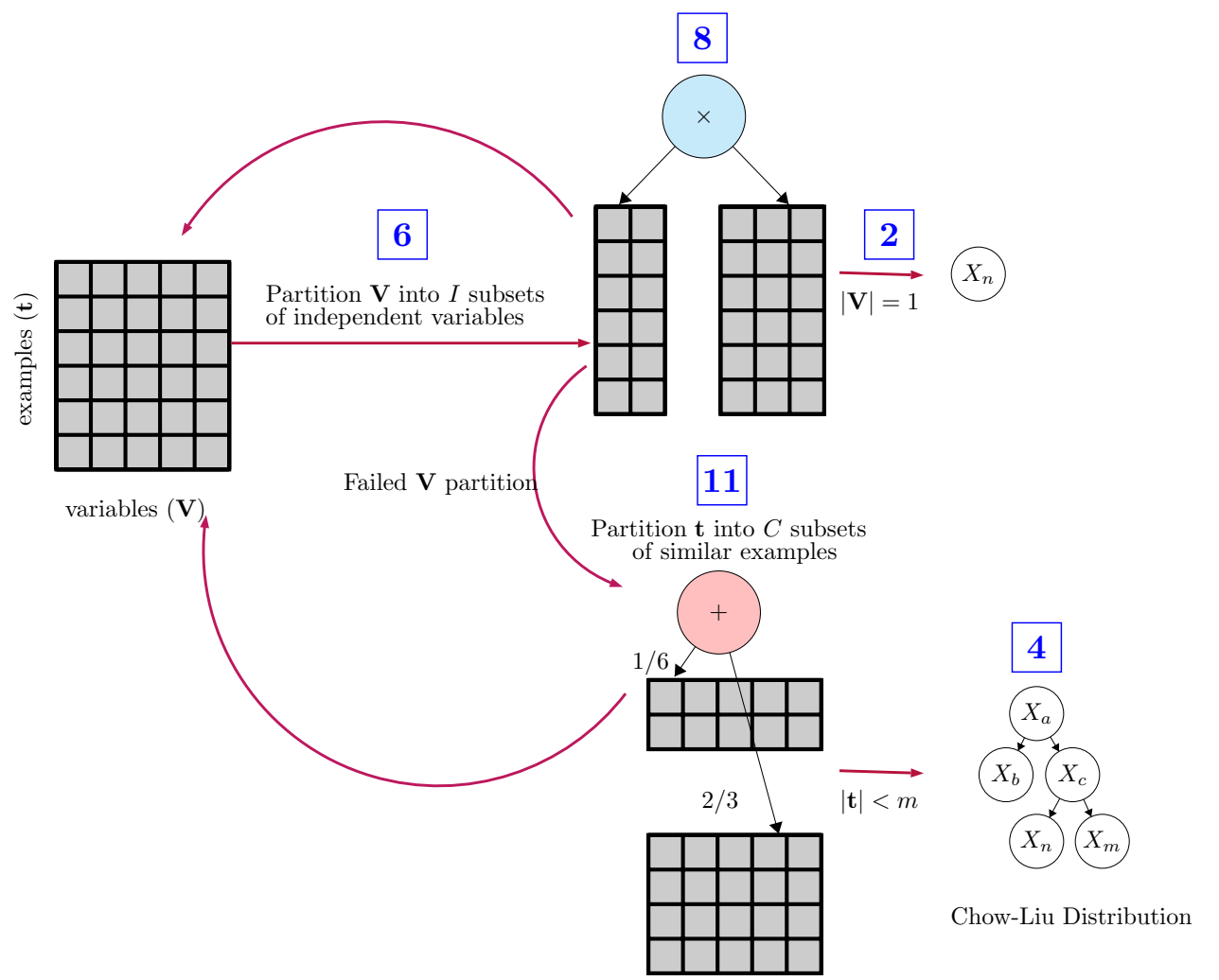

Figure 2.7: Algorithm LearnSPN limiting node splits and Chow-Liu distributions, instead of Naive Factorizations, as leaves. The numbers in blue rectangles represent the associate line number in the Algorithm 2.

\subsubsection{Learning with Direct and Indirect Variable Interactions (ID)}

LearnSPN (or LL or LC) captures variable interactions indirectly using bottom-up clustering to find mixtures. In contrast, most work on learning graphical models (e.g., Bayesian networks or Markov Networks), has focused on finding direct interactions among variables.

\section{Learning Markov Networks with Arithmetic Circuits (ACMN)}

A Markov Network (MN) represents a probability distribution over $\mathbf{V}$ as a normalized product of factors (potentials).

$$
\mathcal{P}_{\mathcal{M}}(\mathbf{V})=\frac{1}{Z} \prod_{c} \phi_{c}\left(\mathbf{D}_{c}\right) .
$$

where each $\phi_{c}$ is a non-negative real-value function, $\mathbf{D}_{c} \subset \mathbf{V}$ represents the factor domain $c$, and $Z$ is the partition function. The distribution also can be represented by equivalent log-linear model:

$$
\log \mathcal{P}_{\mathcal{M}}(\mathbf{V})=\sum_{i} w_{i} f_{i}\left(\mathbf{D}_{i}\right)-\log Z
$$

where $f_{i}$ is a real-value feature function with domain $\mathbf{D}_{i}$ and, $w_{i}$ is a real-value weight. $f_{i}$ can be given by a logical conjunction of variables evaluated as 1 if the expression is satisfied and 0 otherwise, for example $f_{1}\left(V_{1}, V_{3}, V_{8}\right)=v_{1} \wedge \bar{v}_{3} \wedge v_{8}$.

An Arithmetic Circuit (AC) is a rooted, directed acyclic graph, whose interior nodes are sums and products, and its leaves contain parameters (numerical values) or indicator variables. The evaluating or differentiating of an $\mathrm{AC}$ with or without evidence can be done in linear time in the 
size of the circuit [LR13]. The Figure 2.8 shows an example of AC that encodes a two variables Markov Network.

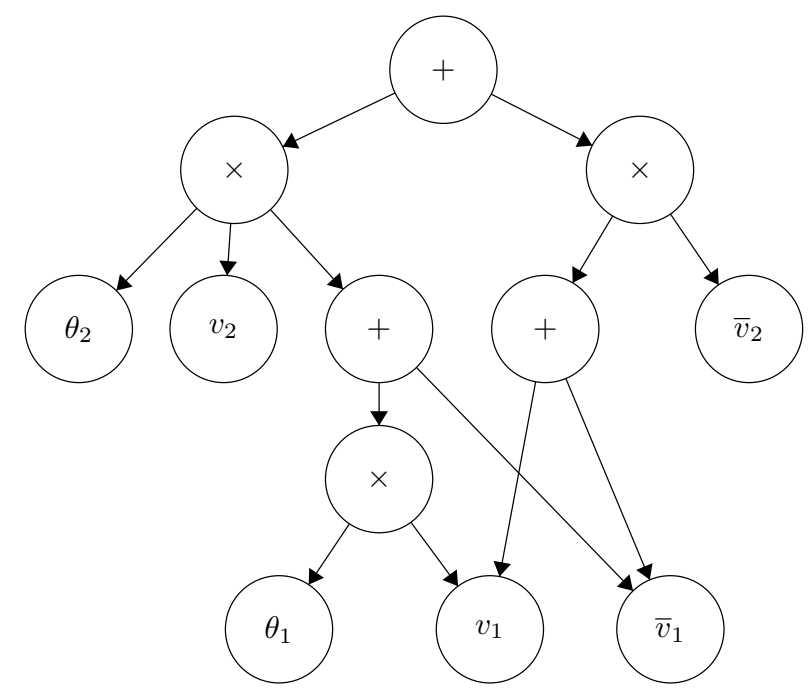

Figure 2.8: Arithmetic Circuit example that encodes a Markov Network with two variables, where $\theta_{1}$ and $\theta_{2}$ are parameters and $v_{1}, \bar{v}_{1}, v_{2}$ and $\bar{v}_{2}$ are indicator functions of variables $V_{1}$ and $V_{2}$

The algorithm ACMN executes a greedy search through the structure space, generating an arithmetic circuit representing a tractable Markov Network [LR13]. The initial structure is the set of all single-variable feature functions. The split operation consists of selecting a feature in the MN, $f$, and combine it with another variable, $V$, creating two new features: $f \wedge v$ and $f \wedge \bar{v}$. Splits are scored according to their effect on the log-likelihood of the MN and the size of the corresponding Arithmetic Circuit (AC):

$$
\operatorname{score}(s)=\Delta_{l l}(s)-\gamma \Delta_{e}(s) .
$$

where $\Delta_{l l}(s)$ is a measure of how much the split $s$ will increase the log-likelihood, $\Delta_{e}(s)$ denotes the number of edges that would be added to the $\mathrm{AC}$ if this split were included. $\gamma$ determines the relative weightings of the two terms.

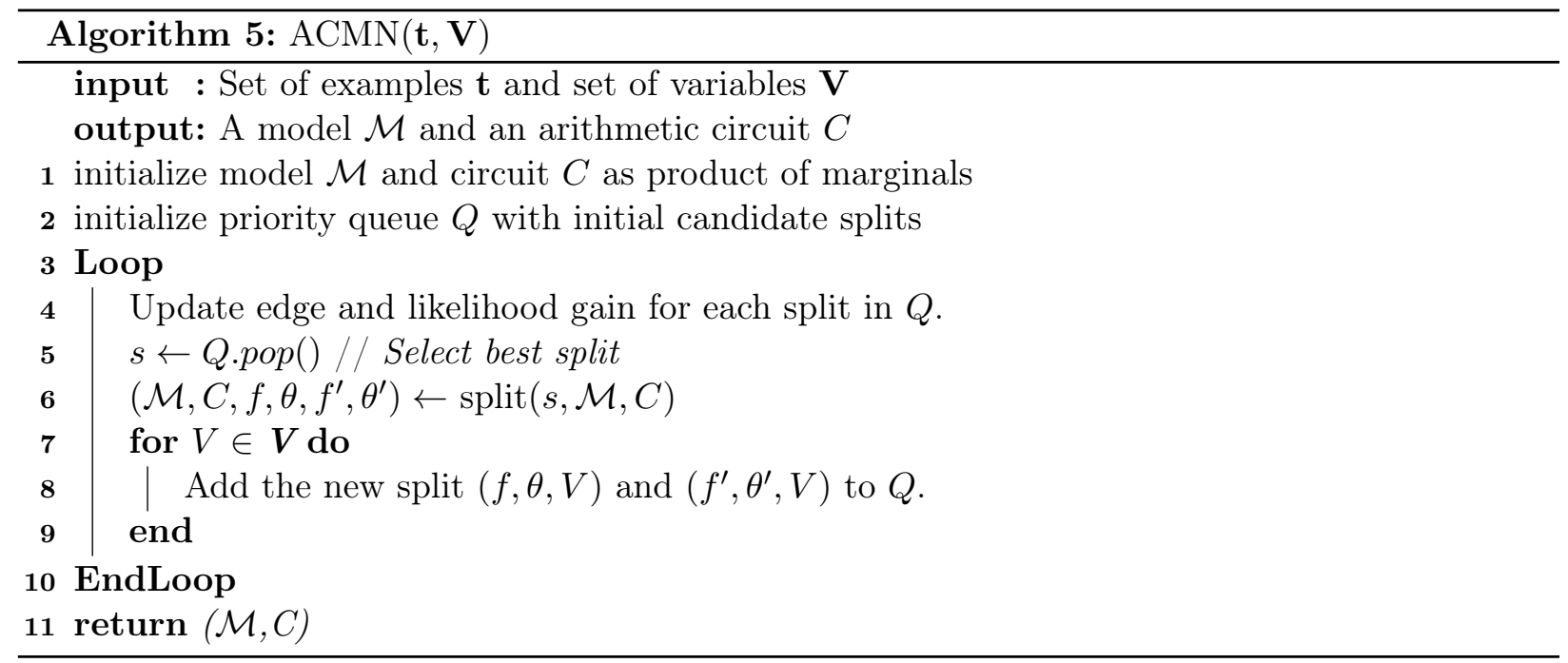




\section{SPN Learning Direct and Indirect Variable Interactions}

SPN Learning Direct and Indirect Variable Interactions (ID) [RL14] unifies these two approaches by learning an SPN and an MN from the same dataset and then selecting the one that best represents the data (maximizes the likelihood). The Figure 2.9 shows an Sum-Product network with arithmetic circuits as leaves learned with the ID algorithm).

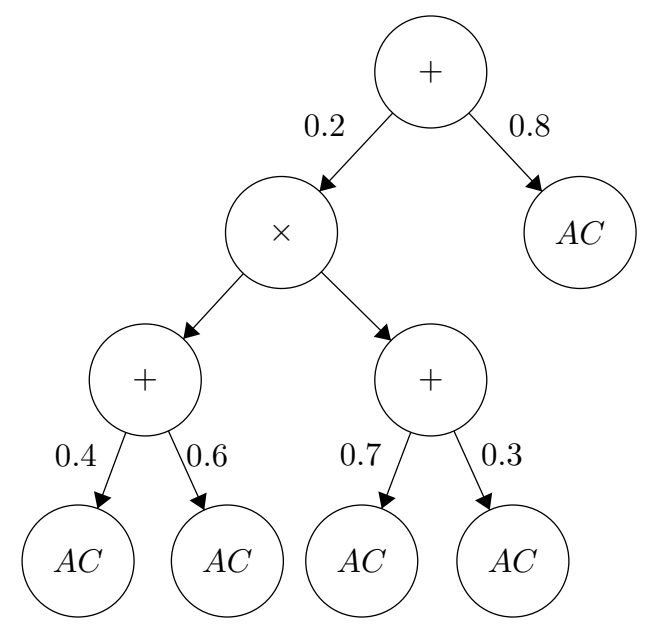

Figure 2.9: Sum-Product network with Markov networks, represented by arithmetic circuits, as leaves

The ID algorithm learns simultaneously at each execution of extend operation an MN, represented by an AC and an SPN with ACs as leaves using the same dataset, and then selects the structure with highest likelihood (the number of times that it executes the extend operation is defined by the user). If the algorithm never selects an AC, the final model is SPN with univariate distribution as leaves. In the other hand if the algorithm selects the $\mathrm{AC}$ in the first extend operation and can not find a structure with better likelihood, the final model is a Markov network. The Algorithms 6, 7 summarize the ID algorithm and the extend operation, and the Figure 2.10 illustrates the Algorithm 7.

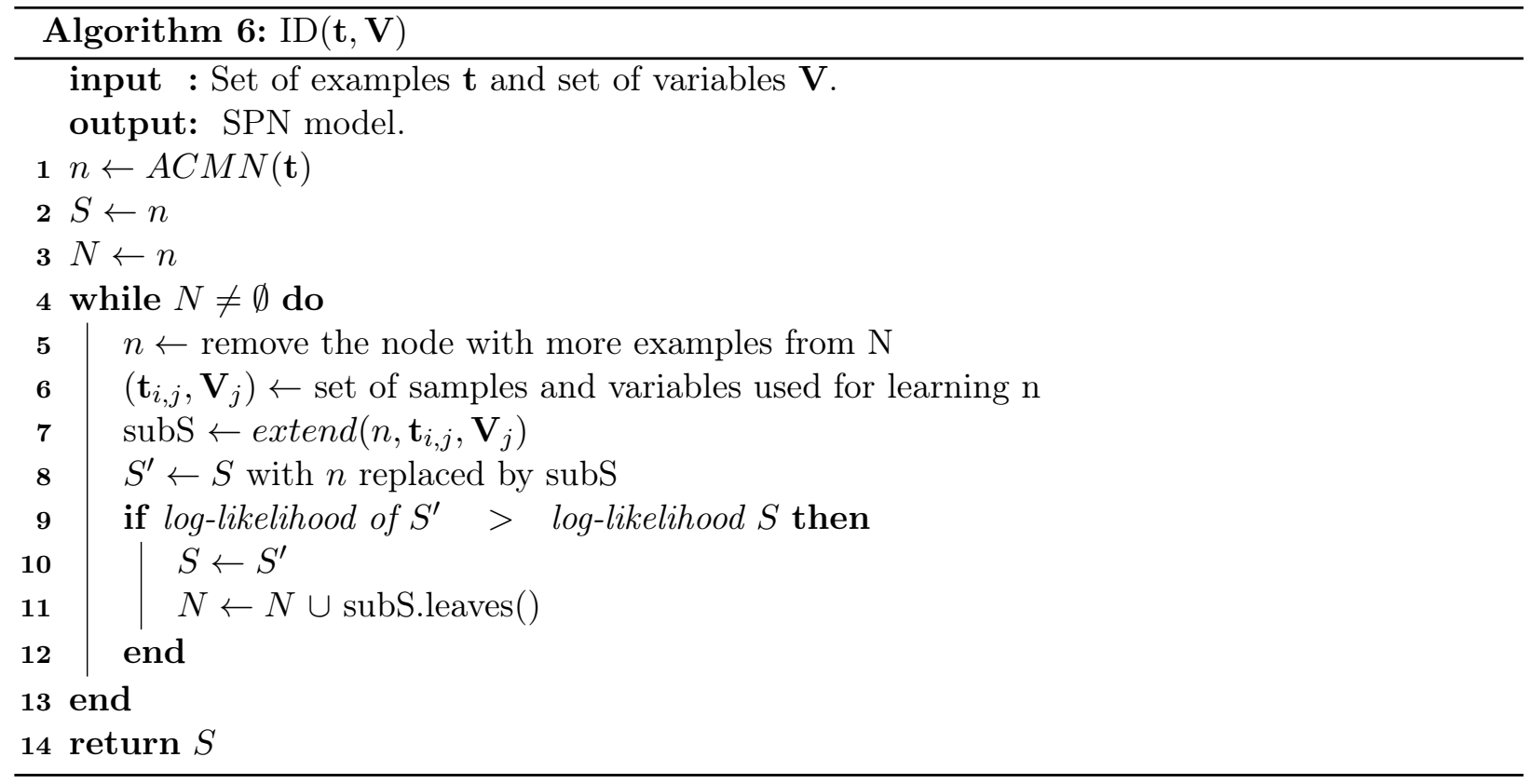




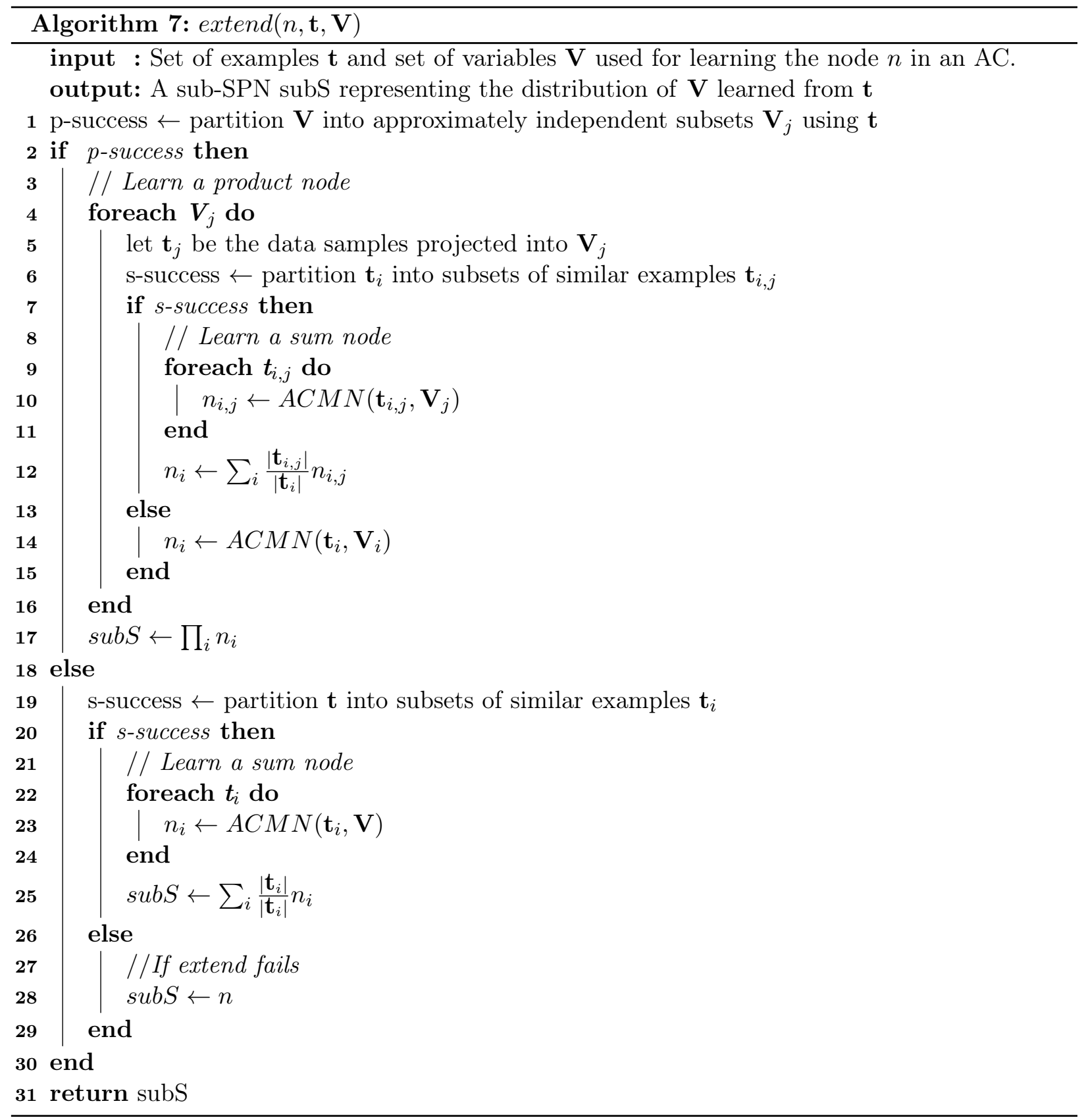




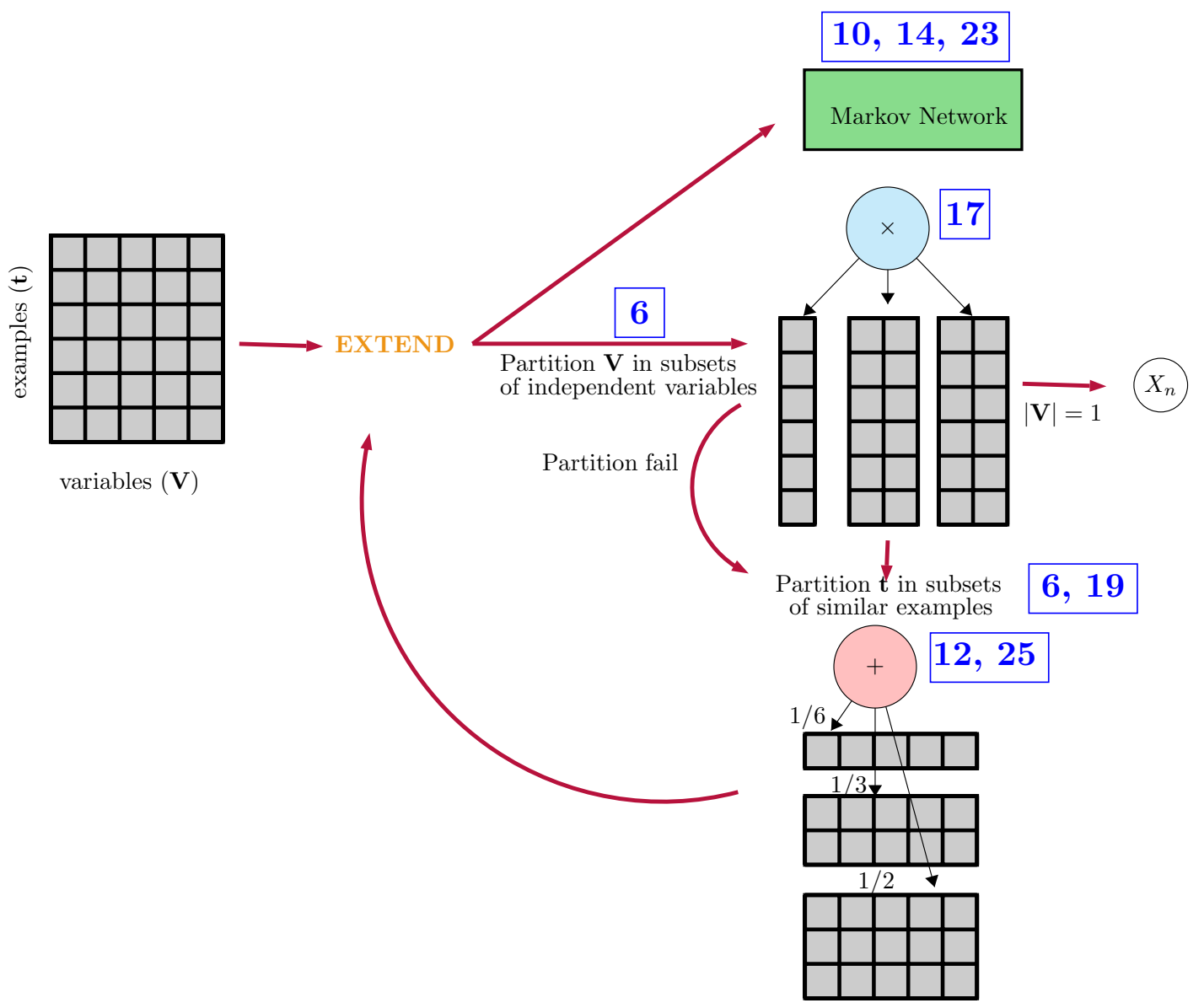

Figure 2.10: Sum-Product network with Markov networks, represented by arithmetic circuits, as leaves. The numbers in blue rectangles represent the associate lines number in the Algorithm 7 . 


\section{Chapter 3}

\section{Multi-Label Classification}

Let $\mathcal{D}$ be a training set with $\mathrm{N}$ examples $\left(\mathbf{x}^{i}, \mathbf{y}^{i}\right)$, where each $\mathbf{x}^{i}=\left(x_{1}^{i}, x_{2}^{i}, \ldots, x_{M}^{i}\right)$ represents a vector of real numbers called feature vector and $\mathbf{y}^{i}=\left(y_{1}^{i}, y_{2}^{i}, \ldots, y_{R}^{i}\right)$ is a Boolean vector representing the relevance labels vector. A coordinate $y_{j}^{i}=1$ indicates that the $j$ th label is present/relevant, while a coordinate $y_{j}^{i}=0$ indicates that the $j$ th label is absent/irrelevant. The Figure 3.1 illustrates the representation described for Multi-Label datasets.

\begin{tabular}{|cccc|cccc|}
\hline \multicolumn{5}{|c|}{$\mathbf{x}$} & \multicolumn{5}{|c|}{$\mathbf{y}$} \\
\hline$x_{1}^{1}$ & $x_{2}^{1}$ & $\cdots$ & $x_{M}^{1}$ & $y_{1}^{1}$ & $y_{2}^{1}$ & $\cdots$ & $y_{R}^{1}$ \\
\hline$x_{1}^{2}$ & $x_{2}^{2}$ & $\cdots$ & $x_{M}^{2}$ & $y_{1}^{2}$ & $y_{2}^{2}$ & $\cdots$ & $y_{R}^{2}$ \\
\hline$\vdots$ & $\vdots$ & $\ddots$ & $\vdots$ & $\vdots$ & $\vdots$ & $\ddots$ & $\vdots$ \\
\hline$x_{1}^{N}$ & $x_{2}^{N}$ & $\cdots$ & $x_{M}^{N}$ & $y_{1}^{N}$ & $y_{2}^{N}$ & $\cdots$ & $y_{R}^{N}$ \\
\hline
\end{tabular}

Figure 3.1: Multi-Label Dataset representation, $\boldsymbol{x}$ denotes the feature vector and $\boldsymbol{y}$ the relevance labels vector

The goal of Multi-Label Classification (MLC) is to obtain a classifier $\mathcal{C}$ using $\mathcal{D}$ that, given a feature vector $\mathbf{x}$, returns a relevance labels vector $\mathbf{y}=\left(y_{1}, \ldots, y_{R}\right)$.

MLC generalizes traditional classification as follows. If for each example only one coordinate is present (i.e., $y_{j}^{i}=1$ for $1 \leq j \leq R$ ), the problem is called a single-label (multi-class) classification (MCC). If $R=1$, the problem is called binary (single-label) classification (BC). The Figure 3.2 shows examples for MLC, MCC and BC.
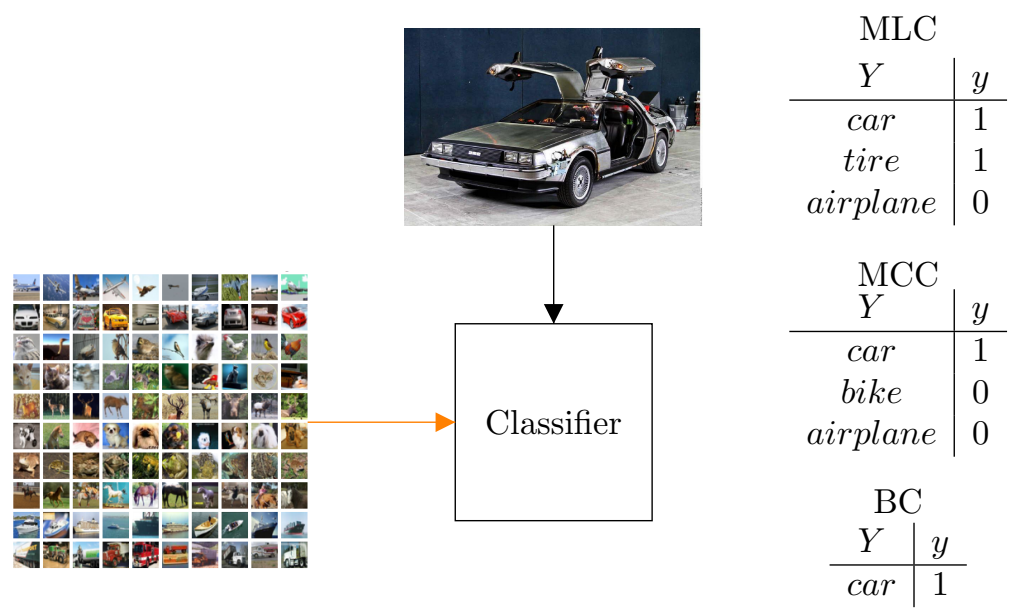

Figure 3.2: Examples of Traditional (binary), Multi-Class and Multi-Label Classifications represented in the tables on the right side $B C, M C C$ and $M L C$ 


\subsection{Methods for Multi-Label Classification}

\subsubsection{Binary Relevance}

The Binary Relevance method (BR) or One-vs-All, consist of decomposing a MLC problem into several binary classification problems [CMM11]. Initially, BR transforms the original Multi-Label training dataset $\mathcal{D}$ in $R$ subsets $\mathcal{D}_{j}$, where each $\mathcal{D}_{j}$ contains all the examples $\left(\mathbf{x}^{i}, y_{j}^{i}\right)$ from the original dataset. The Figure 3.3 shows a training set transformation for three labels.

\begin{tabular}{|cccc|c|c|c|}
\hline \multicolumn{4}{|c|}{$\mathbf{x}$} & \multicolumn{3}{c|}{$\mathbf{y}$} \\
\hline$x_{1}^{1}$ & $x_{2}^{1}$ & $\cdots$ & $x_{M}^{1}$ & $y_{1}^{1}$ & $y_{2}^{1}$ & $y_{3}^{1}$ \\
\hline$x_{1}^{2}$ & $x_{2}^{2}$ & $\cdots$ & $x_{M}^{2}$ & $y_{1}^{2}$ & $y_{2}^{2}$ & $y_{3}^{2}$ \\
\hline$\vdots$ & $\vdots$ & $\ddots$ & $\vdots$ & $\vdots$ & $\vdots$ & $\vdots$ \\
\hline$x_{1}^{N}$ & $x_{2}^{N}$ & $\cdots$ & $x_{M}^{N}$ & $y_{1}^{N}$ & $y_{2}^{N}$ & $y_{3}^{N}$ \\
\hline
\end{tabular}

(a)

\begin{tabular}{|c|c|}
\hline $\mathbf{x}$ & $y_{1}$ \\
\hline $\mathbf{x}^{1}$ & $y_{1}^{1}$ \\
$\mathbf{x}^{2}$ & $y_{1}^{2}$ \\
$\vdots$ & $\vdots$ \\
$\mathbf{x}^{N}$ & $y_{1}^{N}$ \\
\hline
\end{tabular}

(b)

\begin{tabular}{|c|c|}
\hline $\mathbf{x}$ & $y_{2}$ \\
\hline $\mathbf{x}^{1}$ & $y_{2}^{1}$ \\
$\mathbf{x}^{2}$ & $y_{2}^{2}$ \\
$\vdots$ & $\vdots$ \\
$\mathbf{x}^{N}$ & $y_{2}^{N}$ \\
\hline
\end{tabular}

(c)

\begin{tabular}{|c|c|}
\hline $\mathbf{x}$ & $y_{3}$ \\
\hline $\mathbf{x}^{1}$ & $y_{3}^{1}$ \\
$\mathbf{x}^{2}$ & $y_{3}^{2}$ \\
$\vdots$ & $\vdots$ \\
$\mathbf{x}^{N}$ & $y_{3}^{N}$ \\
\hline
\end{tabular}

(d)

Figure 3.3: Binary Relevance transformation on the training dataset with three labels at left 3.3a, generating the following training subsets: $\mathcal{D}_{1} 3.3 b, \mathcal{D}_{2} 3.3 \mathrm{c}$ and $\mathcal{D}_{3} 3.3 d$.

After the training dataset transformation, a binary classifier $\mathcal{C}_{j}(\mathbf{x})$ is generated using each training subset $\mathcal{D}_{j}$, obtaining a set of $R$ classifiers defined by:

$$
\mathcal{C}_{B R}(\mathbf{x})=\left\{j: \mathcal{C}_{j}(\mathbf{x}) \in\{0,1\}\right\}
$$

Finally, the Multi-Label classification is obtained concatenating the predictions of each classifier in $\mathcal{C}_{B R}$

The complexity of generating a Multi-Label classifier with BR method is $O(R \mathcal{C})$, since the generation of each binary classifier consumes time $O(\mathcal{C})$. Thus, applying this method when the number of labels is large, have a high computational cost. Another BR disadvantage, is that not consider the possible dependencies that exist between the labels because it builds independent binary classifiers for each label.

\subsubsection{Chain Classifier}

The Chain Classifier method (CC) is based on BR, but overcomes the independence labels assumption [RPHF11].

Let $\theta$ be an ordering on the labels, the training dataset transformation also creates $R$ subsets denoted by $\mathcal{D}_{\theta_{j}}$. Every subset is composed of examples $\left(\mathbf{x}^{i}, \mathbf{y}_{\theta_{1}}^{i}, \ldots \mathbf{y}_{\theta_{j}}^{i}\right)$, where $\mathbf{y}_{\theta_{1}}^{i}, \ldots \mathbf{y}_{\theta_{j-1}}^{i}$ are part of the feature vector. The Figure 3.4 shows the transformation for a training dataset with three labels.

\begin{tabular}{|c|c|}
\hline $\mathbf{x}$ & $y_{\theta_{1}}$ \\
\hline $\mathbf{x}^{1}$ & $y_{\theta_{1}}^{1}$ \\
$\mathbf{x}^{2}$ & $y_{\theta_{1}}^{2}$ \\
$\vdots$ & $\vdots$ \\
$\mathbf{x}^{N}$ & $y_{\theta_{1}}^{N}$ \\
\hline
\end{tabular}

(a)

\begin{tabular}{|c|c|}
\hline $\mathbf{x}$ & $y_{\theta_{2}}$ \\
\hline$\left\{\mathbf{x}_{1}, y_{\theta_{1}}^{1}\right\}$ & $y_{\theta_{2}}^{1}$ \\
$\left\{\mathbf{x}_{2}, y_{\theta_{1}}^{2}\right\}$ & $y_{\theta_{2}}^{2}$ \\
$\vdots$ & $\vdots$ \\
$\left\{\mathbf{x}_{N}, y_{\theta_{1}}^{N}\right\}$ & $y_{\theta_{2}}^{N}$ \\
\hline
\end{tabular}

(b)

\begin{tabular}{|c|c|}
\hline $\mathbf{x}$ & $y_{\theta_{3}}$ \\
\hline$\left\{\mathbf{x}_{1}, y_{\theta_{1}}^{1}, y_{\theta_{2}}^{1}\right\}$ & $y_{\theta_{3}}^{1}$ \\
$\left\{\mathbf{x}_{2}, y_{\theta_{1}}^{2}, y_{\theta_{2}}^{2}\right\}$ & $y_{\theta_{3}}^{2}$ \\
$\vdots$ & $\vdots$ \\
$\left\{\mathbf{x}_{N}, y_{\theta_{1}}^{N}, y_{\theta_{2}}^{N}\right\}$ & $y_{\theta_{3}}^{N}$ \\
\hline
\end{tabular}

(c)

Figure 3.4: Chain Classifier transformation on the training dataset with three labels in the Figure 3.3a, generating the following train subsets: $\mathcal{D}_{\theta_{1}} 3.4 a, \mathcal{D}_{\theta_{2}} 3.4 b$ and $\mathcal{D}_{\theta_{3}} 3.4 \mathrm{c}$. 
Similar to BR, the Chain Classifier method learns a set of $R$ Binary Classifiers, every $\mathcal{C}_{\theta_{j}}$, learned using the subset $\mathcal{D}_{\theta_{j}}$, subsequently adds the outputs of the previous classifiers $\left\{y_{\theta_{1}}, \ldots, y_{\theta_{j-1}}\right\}$ as new features for the following predictions. It is important to note that the given label order $\theta$ is modeling the relations that might exist between labels in the training dataset. The final Multi-Label classification is given by:

$$
\mathcal{C}_{c c}\left(\mathbf{x}_{i}, \mathbf{y}_{i}\right)=\left\{j: \mathcal{C}_{j}\left(\mathbf{x}, y_{1}, \ldots y_{j-1}\right) \in\{0,1\}\right\}
$$

\subsubsection{Label Power-Set}

The Label Power-Set method (LP) transforms the Multi-Label classification problem into a Multi-Class classification problem [CMM11]. The resulting set of labels in the MCC problem $\left(\mathbf{Y}_{M C C}\right)$ is the set of all possible configurations for the labels in the original MLC problem.

$$
\mathbf{Y}_{M C C}=\operatorname{val}\left(\mathbf{Y}_{M L C}\right)
$$

The LP transforms the training Multi-Label dataset $\mathcal{D}_{M L C}$ into an training Multi-Class dataset $\mathcal{D}_{M C C}$, mapping each feature vector $\mathbf{x}^{i}$ to the corresponding configuration of relevance labels on the original training dataset val $\left(\mathbf{Y}_{M L C}\right)$. Since every label in the original MLC problem is binary, $\left|\mathbf{Y}_{M C C}\right|=2^{R_{M L C}}$. The Figure 3.5 illustrates the LP training set transformation in a dataset with four labels.

\begin{tabular}{|c|c|c|}
\hline $\mathbf{x}$ & $y_{1}$ & $y_{2}$ \\
\hline $\mathbf{x}^{1}$ & 1 & 1 \\
$\mathbf{x}^{2}$ & 0 & 1 \\
$\vdots$ & $\vdots$ & $\vdots$ \\
$\mathbf{x}^{N}$ & 1 & 1 \\
\hline
\end{tabular}

(a)

\begin{tabular}{|c|c|c|c|c|}
\hline $\mathbf{x}$ & 00 & 01 & 10 & 11 \\
\hline $\mathbf{x}^{1}$ & 0 & 0 & 0 & 1 \\
$\mathbf{x}^{2}$ & 0 & 1 & 0 & 0 \\
$\vdots$ & $\vdots$ & $\vdots$ & $\vdots$ & $\vdots$ \\
$\mathbf{x}^{N}$ & 0 & 0 & 0 & 1 \\
\hline
\end{tabular}

(b)

Figure 3.5: Label Power-Set Transformation on a training Multi-Label dataset with three labels 3.5a into a training Multi-Class dataset 3.5b, then $\boldsymbol{Y}_{M L C}=\left\{Y_{1}, Y_{2}\right\}$ and $\boldsymbol{Y}_{M C C}=\{00,01,10,11\}$

Although LP considers label dependence, when it is considered a large or even moderate number of labels, the Multi-Class learning task becomes very costly due to the exponential number of labels. Another important issue is the problem of label imbalance, which happens when there are labels on the training set represented by few examples.

\subsubsection{Probabilistic Graphical Models}

Probabilistic graphical model (PGM) use a graph-based representation as the basis for compactly encoding a complex distribution over a large number of RVs [KF09]. The Figure 3.6 illustrates an probabilistic model (Bayesian Network) example, representing the distribution over six RVs.

A PGM learned from the training dataset $\mathcal{D}$, can be used to predict the relevant labels by computation of the conditional probability of each label $Y_{j}$ given the feature vector $\mathbf{x}$. Or computing the Most Probable Explanation of the labels $\mathbf{Y}$ given the feature vector $\mathbf{x}$.

It is clear that a PGM can be the base classifier for the BR, CC and LP methods. As well as directly perform the MLC by computation of the MPE on the model. Well-known and successful models for classification (Multi-label and Single-label) are, Bayesian Networks, and Markov Networks [CAMG14, ACMG13, FGG97, LMP01]. 


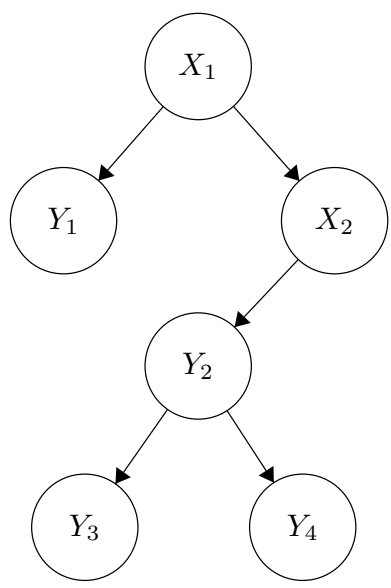

Figure 3.6: An example of a probabilistic model, representing the distributions over six RVs.

The Multi-Label Classifier based on the computation of the Most Probable Explanation on the model is given by the following equation:

$$
\mathcal{C}_{M P E}(\mathbf{x})=\underset{\mathbf{y}}{\operatorname{argmax}} P(\mathbf{y}, \mathbf{x})
$$




\section{Chapter 4}

\section{Multi-Label Classification based on Sum-Product Networks}

In this chapter, we will explain how to use SPN for Multi-Label Classification. There are several approaches for learning a Multi-Label classifier based on SPNs. For example adopting the Binary Relevance approach, for each label $j$, is learned a SPN $\mathcal{S}_{j}$ from the dataset $\mathcal{D}_{j}$ with examples $\left(\mathbf{x}^{i}, \mathbf{y}_{j}^{i}\right)$. A label $j$ is then deemed relevant if:

$$
\mathcal{P}_{\mathcal{S}_{j}}\left(y_{j} \mid \mathbf{x}\right)>1 / 2 \text {. }
$$

The Figure 4.1 illustrates an example for construction and classification procedures of a Multi-Label classifier based en Sum-Product Networks following the Binary Relevance approach, obtained from a dataset with three labels.

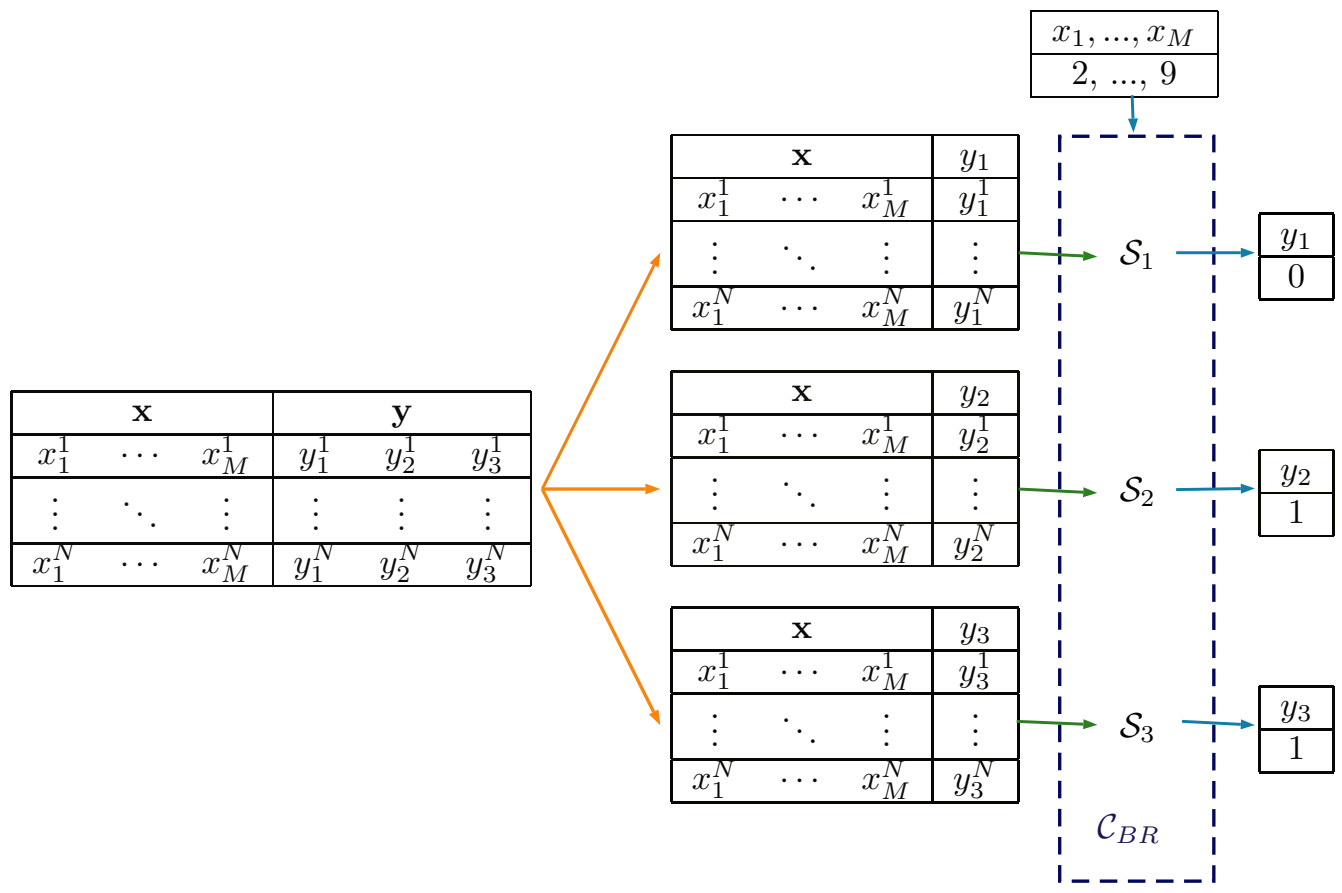

Figure 4.1: Multi-label classifier based on SPN adopting Binary Relevance approach.

An alternative approach to BR is to adopt Classifier Chain, which begins by fixing an ordering for the labels $\theta_{1}, \ldots, \theta_{R}$. Then, similar to BR, learns a SPN for each label as follows, $\mathcal{S}_{\theta_{j}}$ using the dataset $\mathcal{D}_{\theta_{j}}$ which contains examples $\left(\mathbf{x}^{i}, y_{\theta_{1}}^{i}, \ldots, y_{\theta_{j}}^{i}\right)$. The label $j$ is considered relevant if:

$$
\mathcal{P}_{\mathcal{S}_{\theta_{j}}}\left(y_{\theta_{j}} \mid \mathbf{x}, y_{\theta_{1}}, \ldots, y_{\theta_{j-1}}\right)>1 / 2
$$




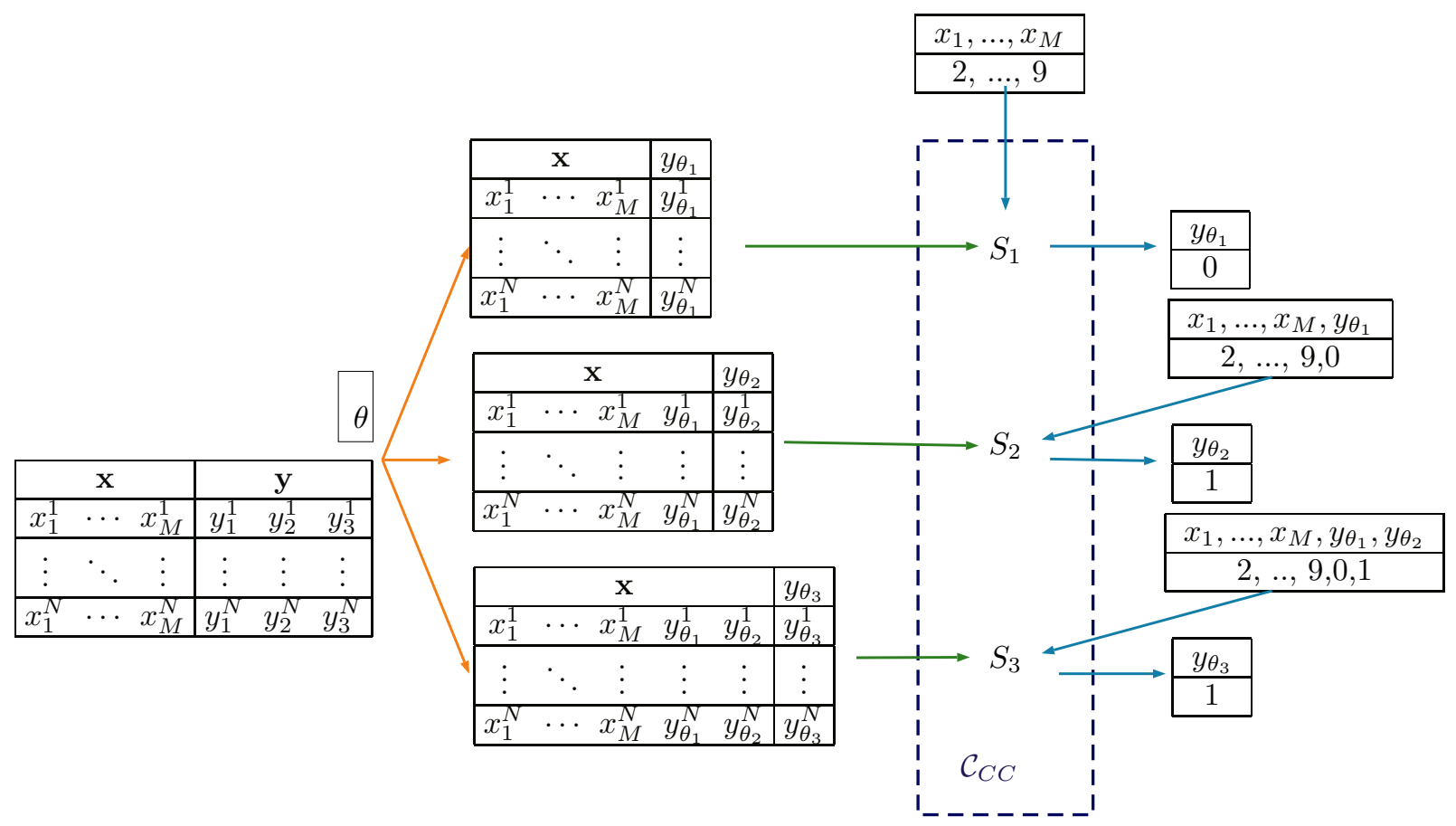

Figure 4.2: Multi-label classifier based on SPN adopting Classifier Chain approach.

As reviewed in section 2.5, SPNs learning is costly. Therefore, follow the BR and CC approaches scales poorly in the number of labels. In fact, in some preliminary results these approaches were not competitive.

We recommend to use the Joint Distribution approach, which learns a single SPN $\mathcal{S}$ over the set of variables $\mathbf{V}$ using the whole training set $\mathcal{D}$, where the scope of $\mathcal{S}$ is the feature vector and labels variables, $\mathbf{V}=\{\mathbf{X}, \mathbf{Y}\}$.

An approach for classification consists in maximizing the marginal probability of each label given the features, nevertheless, the resultant prediction may not be consistent with the data, which indicates that it may have lower joint probability on the model [LI13]. For example, consider the probability distributions for variables $\mathrm{A}$ and $\mathrm{B}$ in Figure 4.3, we want to compute the most likely configuration for $\mathrm{A}$ and $\mathrm{B}$, maximizing the marginals for $\mathrm{A}$ and $\mathrm{B} \operatorname{argmax}_{A} \mathcal{P}(A)=1$ and $\operatorname{argmax}_{B} \mathcal{P}(B)=1$ the configuration obtained is $\mathrm{A}=1$ and $\mathrm{B}=1$, in the other hand computing the $\max$ joint probability we obtain $\operatorname{argmax}_{A, B} \mathcal{P}(A, B)=(0,0)$.

\begin{tabular}{|cc|}
\hline $\mathrm{A}=0$ & $\mathrm{~A}=1$ \\
\hline 0.4 & 0.6 \\
\hline
\end{tabular}

(a)

\begin{tabular}{|c|cc|}
\hline & $\mathrm{B}=0$ & $\mathrm{~B}=1$ \\
\hline $\mathrm{A}=0$ & 0.8 & 0.2 \\
$\mathrm{~A}=1$ & 0.5 & 0.5 \\
\hline
\end{tabular}

(b)

Figure 4.3: Probability distributions of variables $A$ 4.3a and $B$ 4.3b, where $B$ depends on $A$.

In this work, we perform the classification by maximizing the join probability of the relevance label vector given the feature vector:

$$
\mathcal{C}(\mathbf{x})=\underset{\mathbf{y}}{\operatorname{argmax}} \mathcal{P}_{\mathcal{S}}(\mathbf{y} \mid \mathbf{x})
$$

Note that the above equation selects the Most Probable Explanation (MPE) on $\mathcal{S}$. As already 
mentioned in the Section 2.4, this problem is generally NP-hard.

We present some approximate algorithms for MPE to perform Multi-Label Classification, some of which are new.

\subsection{Max-Prod Classification (MC)}

Poon and Domingos suggested finding a MPE of an SPN $\mathcal{S}$ by (i) converting the network into a Max-Product Network $\mathcal{S}^{m}$ by replacing sum nodes with max nodes, then (ii) evaluating the network and finally (iii) backtracking nodes to find the corresponding configuration of the labels nodes [PD11]. The Max-Prod algorithm is described in more detail in section 2.4.

The Figure 4.4 illustrates an example for the Multi-Label classifier using the Max-Prod classification algorithm.

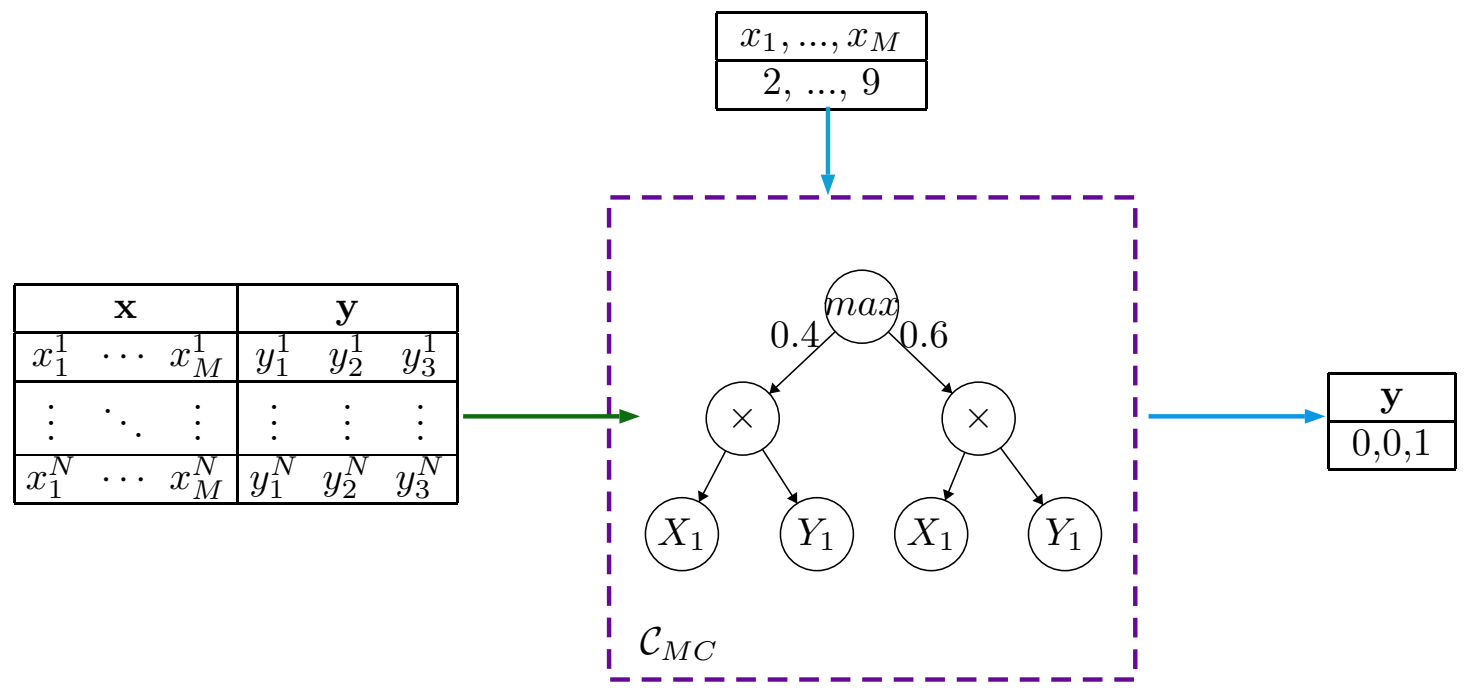

Figure 4.4: Multi-label classifier based on SPN computing the approximation of Most Probable Explanation configuration by Max-Prod Algorithm

The previous algorithm is efficient but inaccurate. We now propose some new algorithms for classification that approximate the most probable explanation .

\subsection{Exhaustive Classification (EC)}

The simplest and least effective algorithm to find $\mathcal{C}(\mathbf{x})$ is a brute-force approach that enumerates all possible relevance label vectors $\left(2^{R}\right.$ combinations of $0 \mathrm{~s}$ and $\left.1 \mathrm{~s}\right)$, computes the probability for each one, by evaluation the SPN, and returns the configuration with the highest probability. Clearly, this algorithm is optimal but does not scale to many labels.

On the other hand, an efficient algorithm selects all distinct relevance label vectors in the training set, and then chooses the prediction with the highest probability as the final classification. However, our classifier loses generality by only predicting relevance label vectors showed in $\mathcal{D}$.

We propose the new algorithm, Exhaustive Classification, that fall between this two extrema. In order to reduce the number of relevance label vectors, selects the set of all distinct predictions on the training set $S V=\left\{\mathbf{y}^{i} \mid \mathbf{y}^{i} \in \mathcal{D}\right\}$ and then optimizes each prediction so as not to lose generality. Given an unlabeled example $\mathbf{x}$, and a relevance label vector $\mathbf{y}^{i}$ such that $\mathbf{y}^{i} \in S V$, EC optimizes the value of each label in $\mathbf{y}^{i}$ to maximizes its joint probability. As result of the optimization of each 
prediction in $S V$ obtains a new set of relevance labels vectors, that we call candidates. The Figure 4.5 shows an example of the optimization process.

Finally, selects the most likely candidate as the prediction associate to $\mathbf{x}$. In summary, Exhaustive

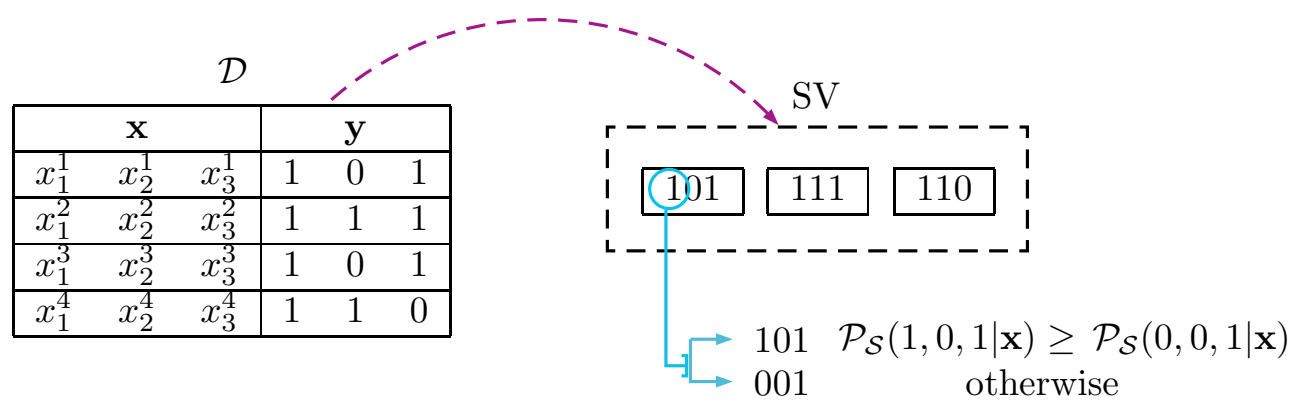

Figure 4.5: Example of Exhaustive classification optimization for a single label in a dataset with three labels

Classification predicts the relevance label vector for $\mathbf{x}$ by:

$$
\mathcal{C}(\mathbf{x})=\max _{\mathbf{y}^{i} \in S V} \underset{y_{j}^{i}}{\operatorname{argmax}} \mathcal{P}_{\mathcal{S}}\left(y_{1}^{i}, \ldots, Y_{j}^{i}=y_{j}^{i}, \ldots y_{R}^{i} \mid \mathbf{x}\right) .
$$

The Algorithm 8 performs the optimization, first in Line 2, the method getDistincPreditions gets all distinct predictions in $\mathcal{D}$, then obtains the set candidates by selecting the relevance for each label that maximizes the conditional probability for the prediction given the feature vector (PC method computes the conditional probability on the SPN).

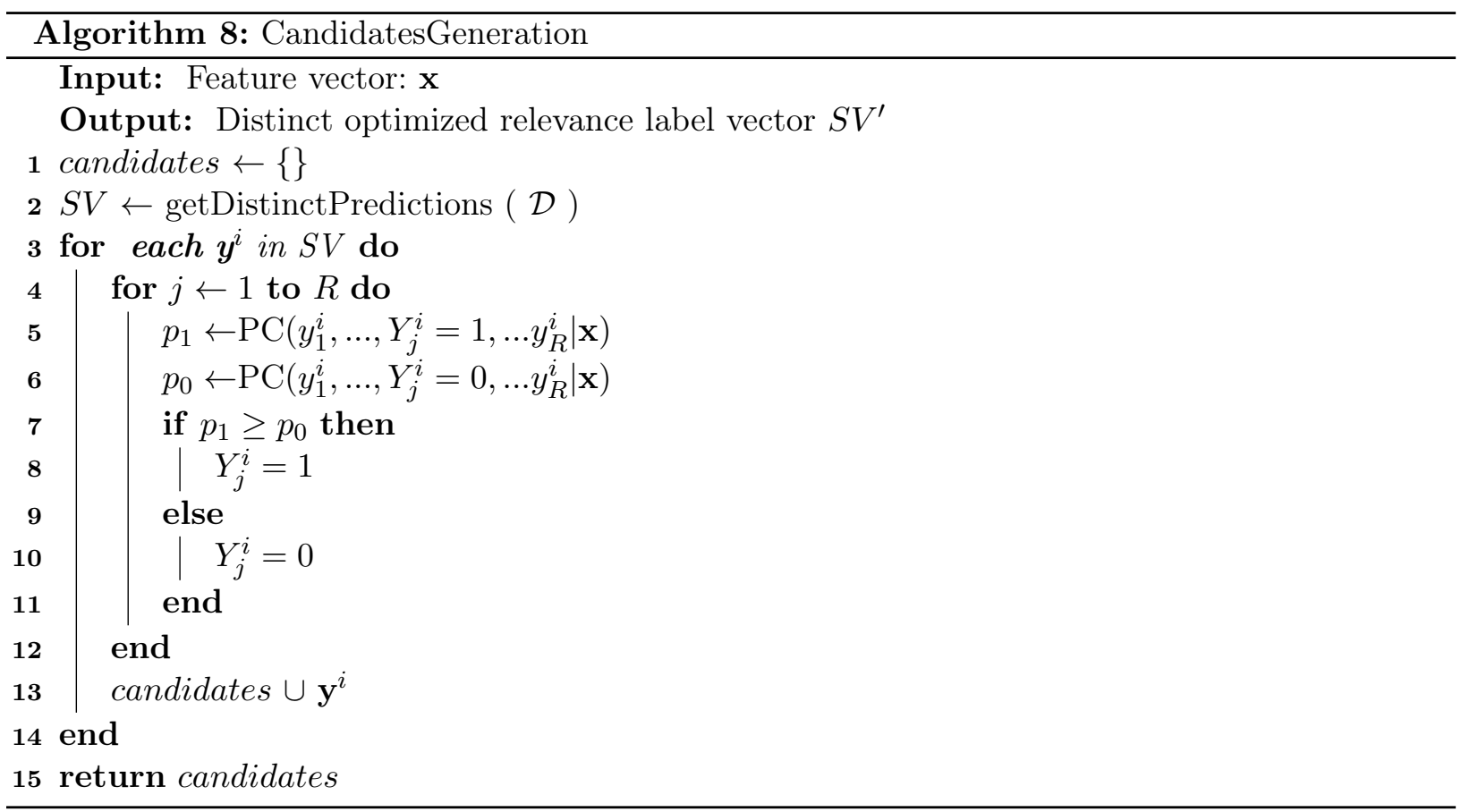

The Algorithm 9 selects the prediction with highest probability from the candidates set as the final classification (P method computes the marginal probability on the SPN). Finally, Figure 4.6 shows an Exhaustive Classification execution example. 

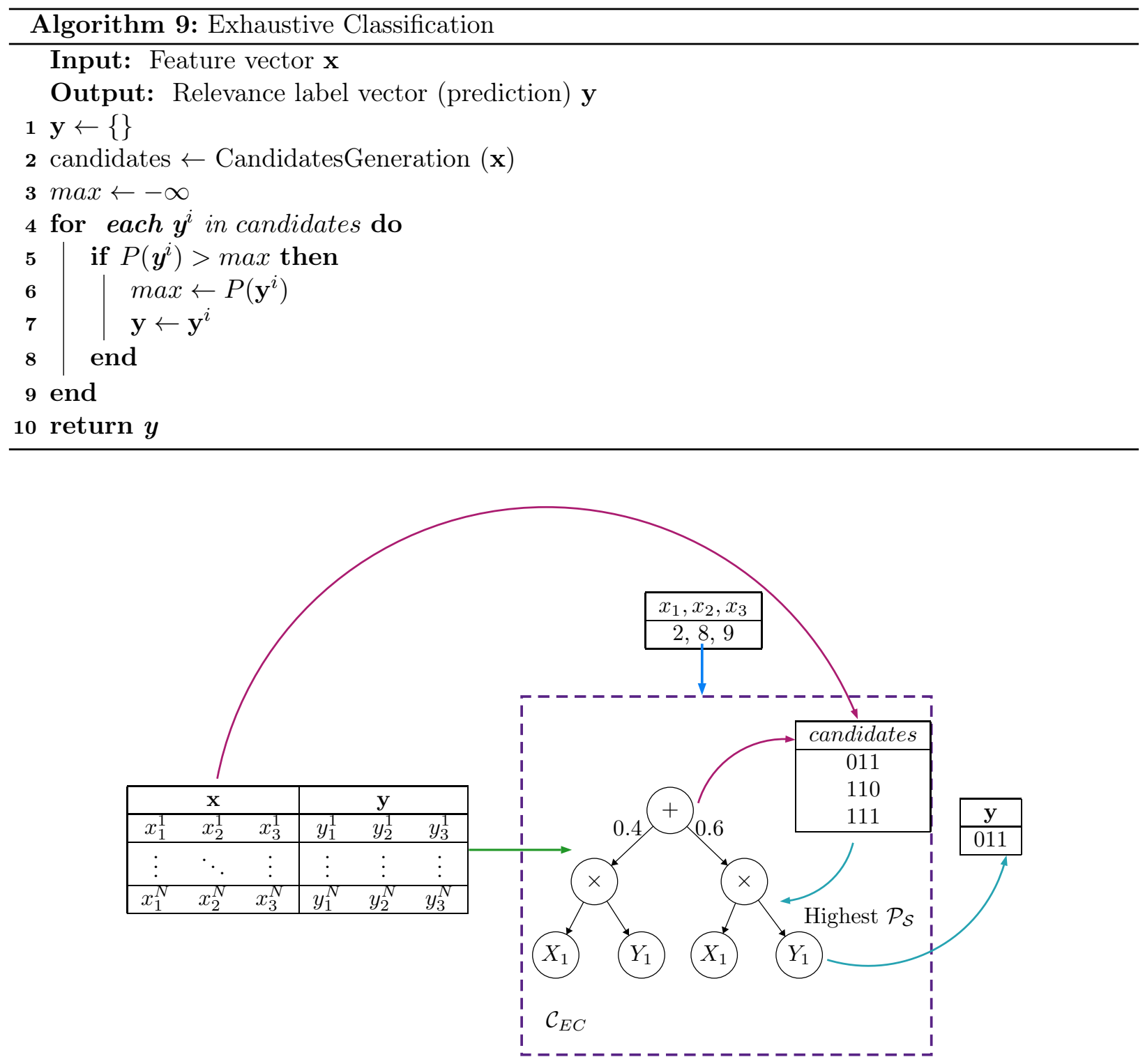

Figure 4.6: Example of Exhaustive Classification in a dataset with three labels, the purple arrows represent the procedure that obtains the candidates, and the cyan arrows represent the classification procedure.

\subsection{Sequential Classification (SC)}

The Sequential Classification algorithm is inspired by the Classifier Chain approach [RPHF11], however, learns a single network. Let $\theta$ be an ordering for the labels. We classify the $\theta_{j}$ th label by:

$$
y_{\theta_{j}}=\underset{y_{\theta_{j}}}{\operatorname{argmax}} \mathcal{P}_{\mathcal{S}}\left(y_{\theta_{j}} \mid \mathbf{x}, y_{\theta_{1}}, \ldots, y_{\theta_{j-1}}\right)
$$

The Algorithm 10 summarizes the Sequential Classification procedure, predicts each label by selecting the value that maximizes the conditional probability of each label relevance given the feature vector and the previous classifications in the ordering $\theta$. The Figure 4.7 shows an example of SC, with a training set with three labels, note that the ordering $\theta$ is used only for the classification. 

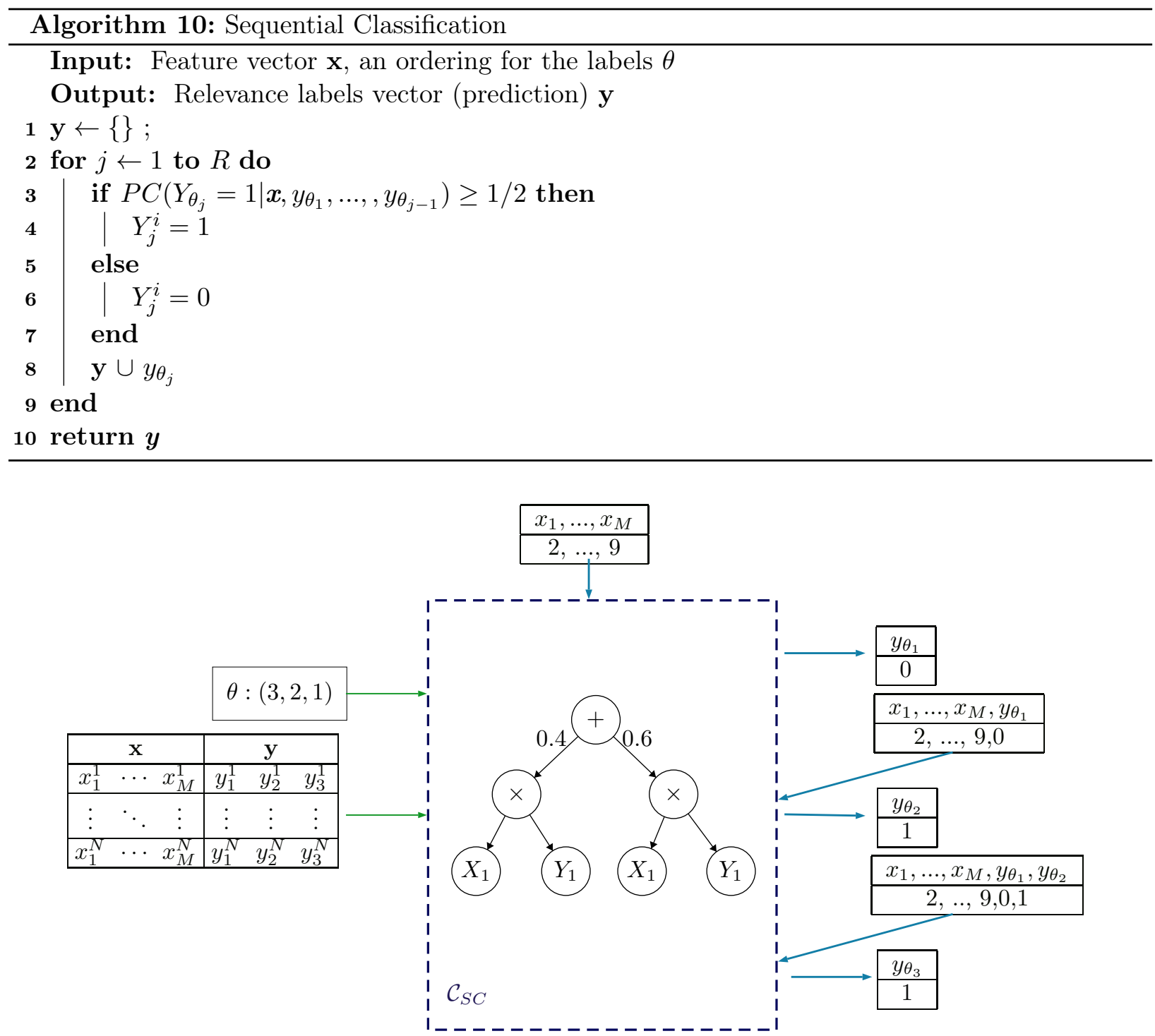

Figure 4.7: An example of sequential classification, with a training dataset with tree labels

Note that unlike CC, a single SPN is used to perform every classification. Like CC, the accuracy of the algorithm is very dependent on the label ordering adopted; also, selecting a good ordering is non trivial. We adopt two different greedy methods for selecting label orderings.

\section{- Static Best-First}

Orders labels by their probability $\mathcal{P}_{\mathcal{S}}\left(Y_{j}=1 \mid \mathbf{x}\right)$ such that $Y_{\theta_{1}}$ has the highest probability, followed by $Y_{\theta_{2}}$, and so on.

\section{- Dynamic Best-First}

Selects an ordering during the classification. According to this method, the $j$ th label to be selected is:

$$
\theta_{j}=\arg \max _{0<k \leq R} \mathcal{P}_{\mathcal{S}}\left(Y_{k}=1 \mid \mathbf{x}, y_{\theta_{1}}, \ldots, y_{\theta_{j-1}}\right),
$$

where $y_{\theta_{1}}, \ldots, y_{\theta_{j-1}}$ are the classifications performed by $\mathrm{SC}$ with the previously selected labels. 


\subsection{Pool of Classifications (PC)}

The previous approaches try to solve the dependency on label ordering by greedily optimizing it. A common alternative approach to cope with sensitivity to model parameters is to consider ensembles of classifications. Let $C S=\left\{\left(y_{1}^{i}, \ldots, y_{R}^{i}\right): i=1, \ldots, k\right\}$ be a set of classifications, our method combine all classification on CS to obtain a single final prediction for the unlabeled example. The Algorithm 11 obtains a set $C S$ of $k$ classifications using the Classify method and then combine them using the method Combine to obtain the final prediction. The Figure 4.8 illustrates an example for $\mathrm{PC}$ with $k=3$.
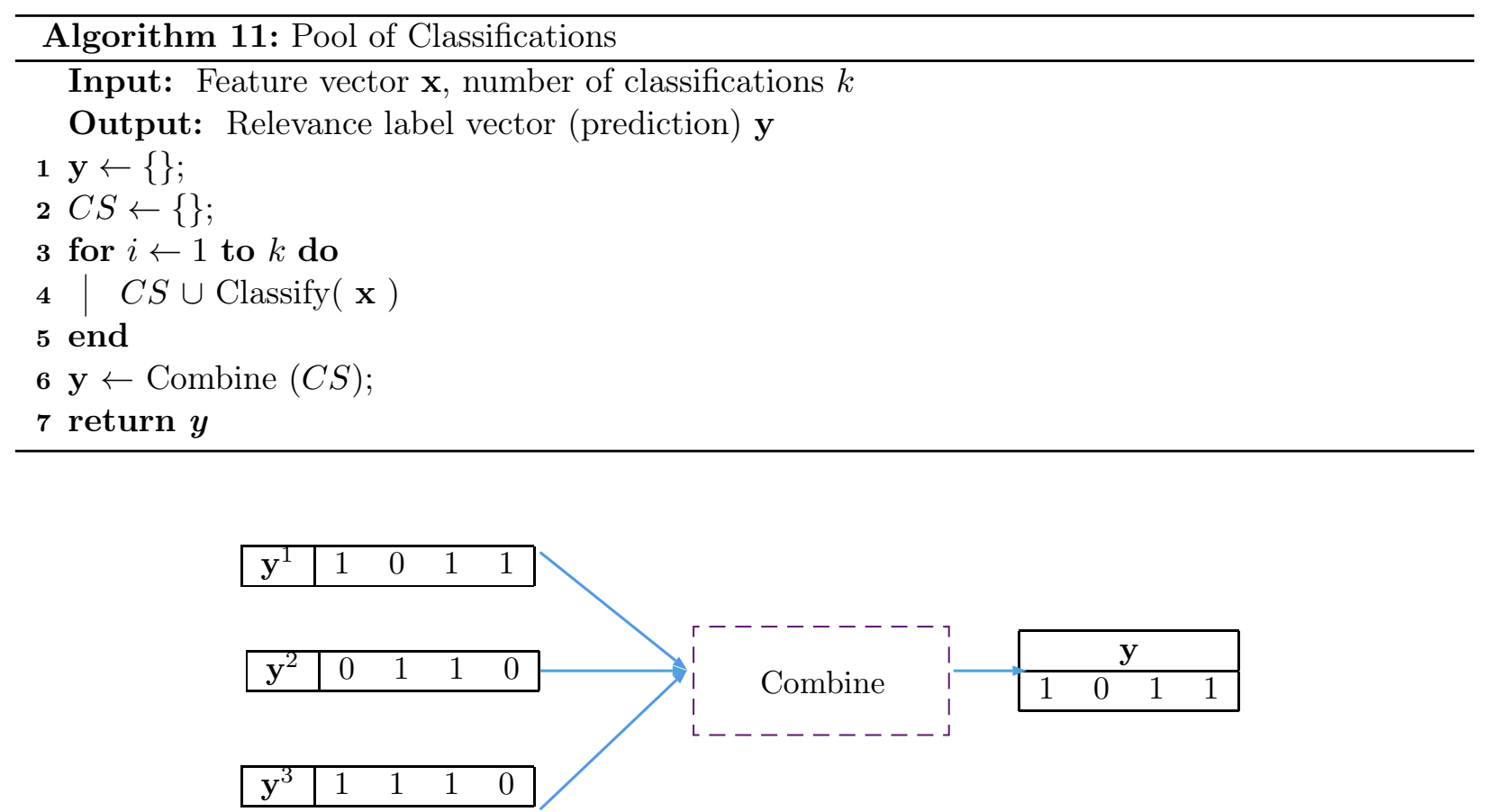

Figure 4.8: An example of pool classification, with four labels and three relevance label vectors

We consider three different methods for producing a single classification from the set $C S$ :

- Majority voting:

Selects labels by comparing weighted, where the weights are formed by votes, that is,

$$
y_{j}=1 \text { iff } \frac{1}{k} \sum_{i=1}^{k} y_{j}^{i} \geq \frac{1}{2} .
$$

- Weighted average :

Obtains the final label classification for each label by:

$$
y_{j}=1 \text { iff } \frac{1}{k} \sum_{i=1}^{k} \mathcal{P}_{\mathcal{S}}\left(Y_{j}^{i}=1 \mid \mathbf{x}\right) \geq \frac{1}{2},
$$

where $\mathcal{P}_{\mathcal{S}}\left(Y_{j}^{i}=1 \mid \mathbf{x}\right)$ is the probability of the label $Y_{j}$ be relevant.

- Max:

It simply selects the ordering whose label achieves the highest joint probability (conditional on the features):

$$
\mathbf{y}=\underset{\mathbf{y}^{i}}{\operatorname{argmax}} \mathcal{P}_{\mathcal{S}}\left(\mathbf{y}^{i} \mid \mathbf{x}\right)
$$


For example, consider the set of classification in the Figure $4.8: \mathbf{y}^{1}=[1,0,1,1] \mathbf{y}^{2}=[0,1,1,0]$ $\mathbf{y}^{3}=[1,1,1,0]$

- Combining with Majority voting method: $y_{1}=1 \quad(2 / 3 \geq 1 / 2), y_{2}=1 \quad(2 / 3 \geq 1 / 2), y_{3}=$ $1(3 / 3 \geq 1 / 2), y_{4}=0 \quad(1 / 3<1 / 2)$ and we obtain the final prediction $\mathbf{y}=[1,1,1,0]$.

- Combining with Weighted average: Consider $\mathcal{P}_{\mathcal{S}}\left(Y_{1}^{1}=1 \mid \mathbf{x}\right)=0.8, \mathcal{P}_{\mathcal{S}}\left(Y_{1}^{2}=1 \mid \mathbf{x}\right)=0.4$, $\mathcal{P}_{\mathcal{S}}\left(Y_{1}^{3}=1 \mid \mathbf{x}\right)=0.7, \mathcal{P}_{\mathcal{S}}\left(Y_{2}^{1}=1 \mid \mathbf{x}\right)=0.1, \mathcal{P}_{\mathcal{S}}\left(Y_{2}^{2}=1 \mid \mathbf{x}\right)=0.6, \mathcal{P}_{\mathcal{S}}\left(Y_{2}^{3}=1 \mid \mathbf{x}\right)=0.7$, $\mathcal{P}_{\mathcal{S}}\left(Y_{3}^{1}=1 \mid \mathbf{x}\right)=0.5, \mathcal{P}_{\mathcal{S}}\left(Y_{3}^{2}=1 \mid \mathbf{x}\right)=0.9, \mathcal{P}_{\mathcal{S}}\left(Y_{3}^{3}=1 \mid \mathbf{x}\right)=0.8, \mathcal{P}_{\mathcal{S}}\left(Y_{4}^{1}=1 \mid \mathbf{x}\right)=0.9$, $\mathcal{P}_{\mathcal{S}}\left(Y_{4}^{2}=1 \mid \mathbf{x}\right)=0.3, \mathcal{P}_{\mathcal{S}}\left(Y_{4}^{3}=1 \mid \mathbf{x}\right)=0.2$.

Then $y_{1}=1 \quad(1.9 / 3 \geq 1 / 2), y_{2}=0 \quad(1.4 / 3<1 / 2), y_{3}=1 \quad(2.3 / 3 \geq 1 / 2), y_{4}=$ $0 \quad(1.4 / 3<1 / 2)$, and we obtain the final prediction $\mathbf{y}=[1,0,1,0]$.

- Combining with Max: Consider $\mathcal{P}_{\mathcal{S}}\left(\mathbf{y}^{1}\right)=0.5, \mathcal{P}_{\mathcal{S}}\left(\mathbf{y}^{2}\right)=0.8, \mathcal{P}_{\mathcal{S}}\left(\mathbf{y}^{3}\right)=0.7$, then $\mathbf{y}=$ $\mathbf{y}^{2} \quad(0.8>0.7>0.5)$ and we obtain the final prediction $\mathbf{y}=[0,1,1,0]$.

We can use several basis methods for classification, in this work we proposed use two methods previously described in this chapter.

\section{Pool of Sequential Classification (PSC)}

Consider a set of label orderings (obtained e.g. by randomly sampling orderings) $\theta^{1}, \ldots, \theta^{k}$, the set $C S$ be a set of classifications obtained from each ordering by using sequential classification.

To obtain the algorithm for PSC we need to add a new line on the Algorithm 11 between the lines (2-3) to generate randomly $k$ orderings for the labels and, modify the line 4 to $C S \cup$ SequentialClassification $\left(\mathbf{x}, \theta^{i}\right)$, therefore we obtain a set of classifications calling SC using distinct orderings. The Figure 4.9 shows an example of Pool Sequential Classification, using a training dataset with two labels.

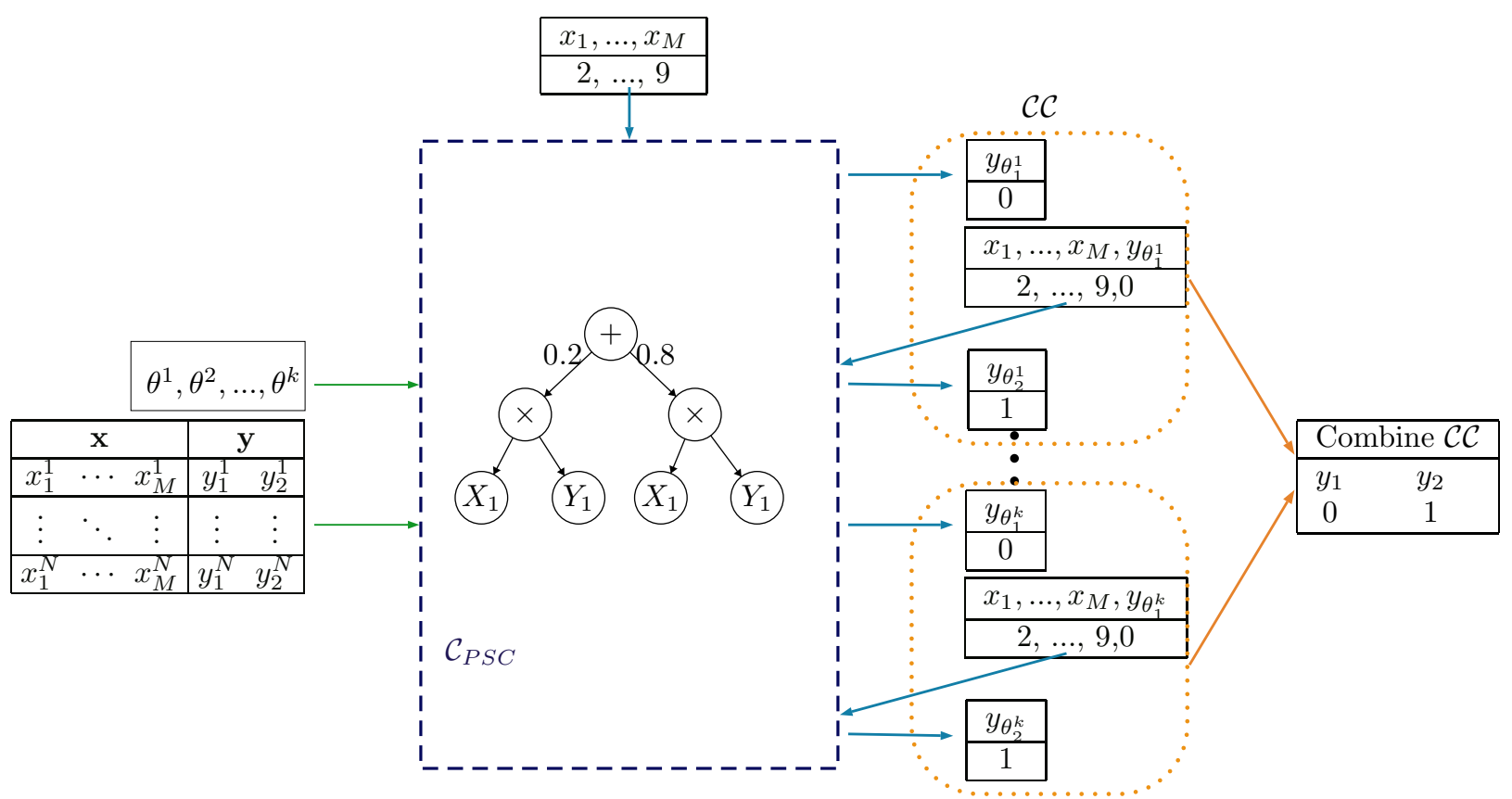

Figure 4.9: An example of Pool Sequential Classification, using a training dataset with two labels 


\section{Pool of Exhaustive Classification (PEC)}

Let candidates be the set obtained by EC optimization, described in the Section 4.2. The set $C S$ is the set of $k$ predictions with highest probability from candidates. If we use the combining method Max, described in the previous subsection, would obtain exactly the same relevance label vector that using only Exhaustive Classification.

To obtain the algorithm for PEC we only call the method SetExhaustiveClassifications $(\mathbf{x})$, that be similar to the Algorithm 9, but instead of returns a single classification $\mathbf{y}$ returns the set of most likely $k$ classification on the model. The Figure 4.10 shows an example of Pool Exhaustive Classification, using a training dataset with two labels.

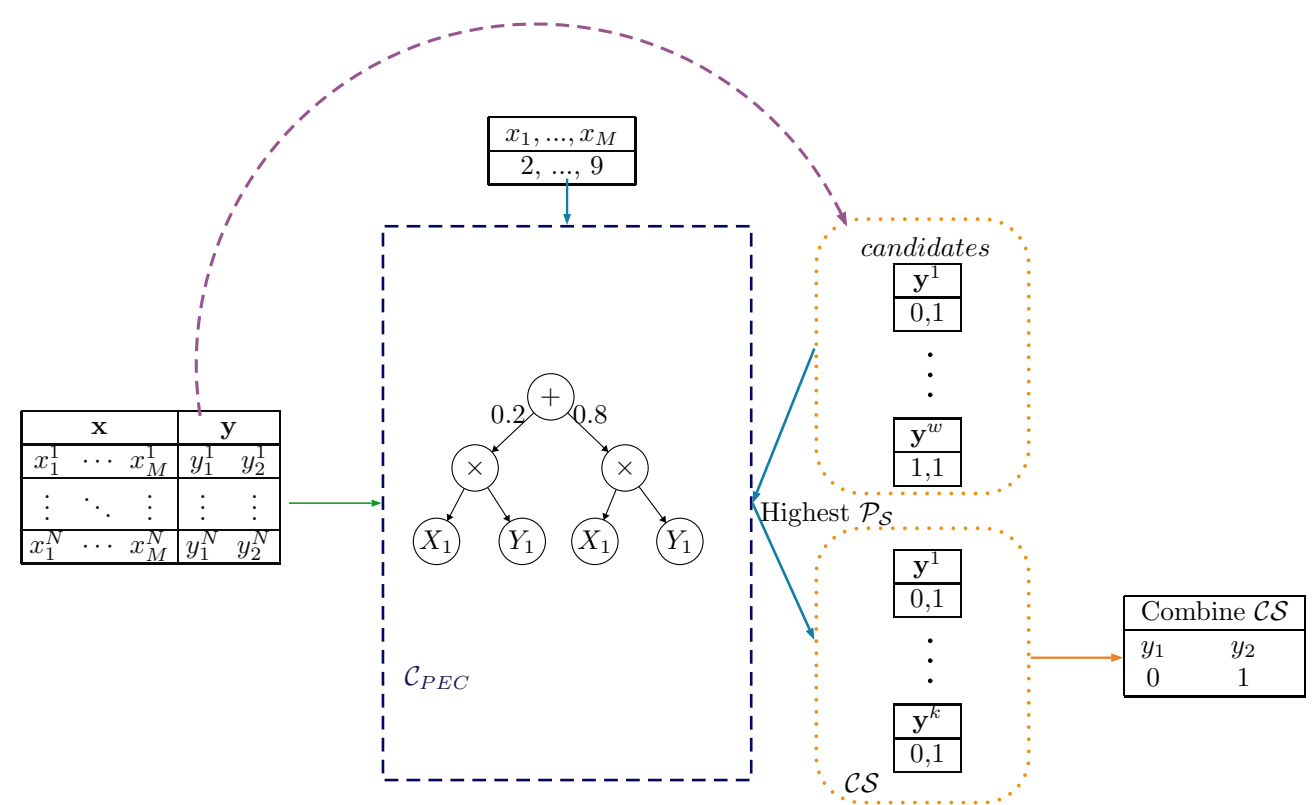

Figure 4.10: An example of Pool Exhaustive Classification, using a training dataset with two labels.

\subsection{Complexity Analysis}

Let $|\mathcal{S}|$ be the number of nodes in $\mathcal{S}$. Exhaustive Classification takes time $O(R N|\mathcal{S}|)$, since it needs to optimize each label at most $N$ relevance labels vectors and compute conditional probabilities in each of them from $\mathcal{S}$. The time complexity of Max-Prod Classification is $O(|\mathcal{S}|)$, since it requires two passes in the network $\mathcal{S}$, each taking linear time in $|\mathcal{S}|$. The time complexity of Sequential Classification depends on the method used to optimize the classification order. Static Best-First takes time $O(R|\mathcal{S}|$ ) to rank the labels (as we need to compute conditional probabilities for each label). Dynamic Best-First takes time $O\left(R^{2}|\mathcal{S}|\right)$ to rank the labels, since at every classification we need to re-rank labels by their conditional probability. In either method, the time to produce classifications is $O(R|\mathcal{S}|)$. Thus, both approaches are linear in the size of the network; Static Best-First is also linear in the number of labels, while Dynamic Best-First is quadratic. In the case of Pool Classifications the complexity for Weighted Average, Voting and Max criteria is k times the complexity time of the basis classification algorithm. The Pool Sequential Classification takes time $O(k R|\mathcal{S}|)$, since it uses random orderings. The time complexity of Pool Exhaustive Classification is $O(k R N|\mathcal{S}|)$. 


\section{Chapter 5}

\section{Experiments}

\subsection{Datasets}

We evaluated our proposed classifiers on 10 benchmark datasets ${ }^{1}$, these datasets were also used for Multi-Label Classification with CutNets [DMVE16].

Since the current implementations of learning algorithms for SPNs work only on binary data, we discretized the numeric features for each dataset by the Label-Attribute Interdependence Maximization (LAIM) [CLGV16].

Table 5.1 shows general characteristics of discretized datasets, such as domain, number of features (M), number of examples (N), number of labels (R), label cardinality $L C(\mathcal{D})=\frac{1}{N} \sum_{i=1}^{N}\left|\mathbf{y}_{i}\right|$ and label density $L D(\mathcal{D})=\frac{1}{N} \sum_{i=1}^{N} \frac{\left|\mathbf{y}_{i}\right|}{R}$, where $|\mathbf{y}|$ returns the number of ones in the binary vector y.

\begin{tabular}{|c|c|c|c|c|c|c|}
\hline Dataset & Domain & $\mathrm{M}$ & $\mathrm{N}$ & $\mathrm{R}$ & $\mathrm{LC}$ & $\mathrm{LD}$ \\
\hline Arts-Yahoo & Text & 500 & 7484 & 26 & 1.653 & 0.063 \\
Business-Yahoo & Text & 500 & 11214 & 30 & 1.598 & 0.053 \\
CAL500 & Music & 68 & 502 & 174 & 26.043 & 0.149 \\
Emotions & Music & 72 & 593 & 6 & 1.868 & 0.311 \\
Flags & Images & 19 & 194 & 7 & 3.391 & 0.484 \\
Health-Yahoo & Text & 500 & 9205 & 32 & 1.644 & 0.051 \\
Human & Biological & 440 & 3106 & 14 & 1.185 & 0.084 \\
Plant & Biological & 440 & 978 & 12 & 1.078 & 0.089 \\
Scene & Images & 294 & 2407 & 6 & 1.073 & 0.178 \\
Yeast & Biological & 103 & 2417 & 14 & 4.237 & 0.301 \\
\hline
\end{tabular}

Table 5.1: Multi-Label Datasets, their domains, size of their feature vector (M), number of instances (N), number of labels (R), label cardinality $(L C)$ and label density (LD)

- Yahoo Datasets: The Arts-Yahoo, Business-Yahoo and Health-Yahoo datasets were obtained from the yahoo.com domain in order to categorize real Web page linked from the "Arts \& Humanities" , "Business \& Economy" and "Health" categories into a number of second-level subcategories [US03].

- CAL500: Music dataset used is a set of 500 "Western popular" songs associate to emotional content, genre, instrumentation, or vocal characterizations [TBTL08]

\footnotetext{
${ }^{1}$ Available at https://github.com/nicoladimauro/dcsn and http://meka.sourceforge.net
} 
- Emotions: The dataset consists of 100 songs from each of the following 7 different genres: Classical, Reggae, Rock, Pop, Hip-Hop, Techno and Jazz labeled with emotions like amazedsurprised or angry-fearful [TTKV08].

- Flags Contains details of some countries and their flags, and the goal is to predict some of the features [CLGV16].

- Human: This dataset is used to predict the sub-cellular locations of proteins according to their sequences on Human species [CLGV16].

- Plant: This dataset is used to predict the sub-cellular locations of proteins according to their sequences, for Plant species [CLGV16].

- Scene: Dataset of images used for categorize images into semantic classes such as beaches, sunsets or parties [BLSB04].

- Yeast The dataset is formed by micro-array expression data and phylogenetic profiles with 2417 genes dataset. Each gene is associated with a set of 14 functional classes like as Ionic Homeostasis or Cellular Biogenesis [EW02].

\subsection{Metrics}

To measure the performance of the proposed classifiers we adopt three commonly used metrics in MLC, each tailored for capturing different characteristics of the predictions [DWCH12, DMVE16].

\subsubsection{Hamming Score (HS)}

Rewards the percentage of correctly predicted labels, and is given by:

$$
H S(\mathcal{D}, \mathcal{C})=\frac{1}{N R} \sum_{i=1}^{N} \sum_{j=1}^{R} \mathbb{I}\left(y_{i, j}=\hat{y}_{i, j}\right) .
$$

HS does not distinguishes between correctly predicted relevance and irrelevance, and can be overoptimistic when the number of labels is high and label density is low.

\subsubsection{Exact Match (EM)}

Rewards classifications by the percentage of examples for which predictions are perfect: the relevant labels as predicted as relevant and the irrelevant one are predicted as absent. It is given by:

$$
E M(\mathcal{D}, \mathcal{C})=\frac{1}{N} \sum_{i=1}^{N} \mathbb{I}\left(\mathbf{y}_{i}=\hat{\mathbf{y}}_{i}\right) .
$$

EM can be over pessimistic, as the failure of a single relevance/irrelevance deems a classification incorrect.

\subsubsection{Accuracy (Acc)}

Rewards relevant predictions while discounting for irrelevant predictions, thus obtaining a compromise between HS and EM. It is computed by:

$$
A c c(\mathcal{D}, \mathcal{C})=\frac{1}{N} \sum_{i=1}^{N} \frac{\left|\mathbf{y}_{i} \wedge \hat{\mathbf{y}}_{i}\right|}{\left|\mathbf{y}_{i} \vee \hat{\mathbf{y}}_{i}\right|}
$$


Where $\mathbb{I}($.$) is the indicator function, \wedge \vee$ are logical operators (AND, OR), $\hat{y}_{i, j}$ is the predicted value of the label $j$ for the example $i$ in the $\mathcal{C}$ classifier and $y_{i, j}$ is the corresponding expected value in the dataset $\mathcal{D}, \hat{\mathbf{y}}_{i}$ is the predicted of the relevance labels vector for the example $i$ in the $\mathcal{C}$ classifier and $\mathbf{y}_{i}$ is the corresponding expected relevance labels vector in the dataset $\mathcal{D}$.

\subsection{Algorithms}

For the experiments, we adopt the Cross Validation method to evaluate the generalization capability of our models, that splits the dataset in 5-folds, then uses 4 folds as training set and the remaining fold as test set, this process is executed 5 times by alternating the test set in a non-repeating manner. The experiments for each Multi-Label Classifiers (RSVM, RCNET and SPN-based) use exactly the same partitions at each execution of Cross Validation.

\subsubsection{Multi-label Classifiers based on SPN}

We obtain different SPN-based classifiers by varying the learning algorithm used (LL, LC or ID) as well as the classification algorithm (EC, MC, SC, P $)^{2}$. We denote each choice by the concatenations of the acronyms of the classification algorithm and learning algorithm. For example, EC+LL denotes the classifiers that uses LL as learning algorithm and EC for classification.

\begin{tabular}{l|ccc}
\hline & LL & LC & ID \\
\hline EC & EC+LL & EC+LL & EC+ID \\
MC & MC + LL & MC +LC & MC +ID \\
SC & SC+LL & SC+LC & SC+ID \\
PSC & PSC+LL & PSC + LC & PSC+ID \\
PEC & PEC + LL & PEC + LC & PEC + ID \\
\hline
\end{tabular}

Table 5.2: Multi-Label Classifiers based on Sum-Product Networks

Some classification algorithms require additional specifications. For example, SC uses either random, Static Best-First or Dynamic Best-First ordering. We randomly generated ten orderings when using Pool Sequential (PS) and selected ten vectors for Pool Exhaustive (PE), then applied the weighted average, majority voting and max methods. In the results Tables for SC, PS and PE, we only show the classifier with better performance using the ordering methods or the combination methods respectively.

We tuning the Sum-Product network learning algorithms, performing a grid-search on the likelihood to select the parameters. The algorithm limiting node splits and the algorithm with multivariate distributions as leaves, tuning G-test factor, Minimum instance number in a slice and smoothing probability factor ${ }^{3}$. While for the algorithm with direct and indirect variable interactions, we used the Libra toolkit [LR15], tuning the Per-split penalty, Max sum nodes cardinalities and Maximum number of node extensions.

\footnotetext{
${ }^{2}$ Available at https://github.com/giulianavll/MLC-SPN

${ }^{3}$ Source code is available at http://www.di.uniba.it/ vergari/code/spyn.html
} 


\subsubsection{State-Of-Art Multi-Label Classifiers}

We compare our approaches against the state-of-the-art Multi-Label classifiers RCNET and RSVM.

\section{RCNET}

RCNET employs CutNets and adopts the exact MPE approach [DMVE16]. ${ }^{4}$. Which was previously shown to outperform several other approaches such as Bayesian Chain Classifier, Binary relevance and the classifier chains methods with base classifiers Naive Bayes and Tree Augmented Naive Bayes.

\section{RSVM}

RSVM adopts the Random k-Labelsets for Multi-Label Classification (RaKel) approach [TKV11] where an ensemble of label power-set classifiers is learned. It uses as Multi-Class classifiers Support Vector Machines with polynomial kernel (using their openly implementation in MEKA ${ }^{5}$ ). Which shown competitive results compared with the classifiers in the experiments of LAIM $^{6}$ (such as Dependent Binary Relevance, Ensemble of Classifier Chains and Hierarchy of Multi-label classifiers approaches with J48 as base classifier).

\subsection{Results and Discussion for Multi-Label Classifiers based on SPN and State-of-art}

Tables 5.3, 5.4 and 5.5 show the performance and ranking for Hamming Score, Exact Match, and Accuracy respectively for our proposed classifiers based on SPNs, the Multi-Label classier based on CutsetNets (RCNET) and the Random k-labelsets approach using as basis classifier Support Vector Machines (RSVM). As we discussed in the previous chapter, the state-of-art classifiers chosen for the experiments (viz. RCNET and RSVM) was previously shown outperform several other approaches.

\begin{tabular}{|c|c|c|c|c|c|c|c|c|c|c|c|}
\hline & Arts & Business & CAL500 & Emotions & Flags & Health & Human & Plants & Scene & Yeast & AvgRnk \\
\hline $\mathrm{SC}+\mathrm{LL}$ & $0.945(\mathbf{2})$ & $0.977(\mathbf{1})$ & $0.862(\mathbf{1})$ & $0.793(8)$ & $0.722(7)$ & $0.968(8)$ & $0.919(\mathbf{1})$ & $0.915(\mathbf{1})$ & $0.899(7)$ & $0.784(2)$ & 3.8 \\
\hline $\mathrm{EC}+\mathrm{ID}$ & $0.943(4)$ & $0.977(\mathbf{1})$ & $0.829(7)$ & $0.808(\mathbf{2})$ & $0.768(2)$ & $0.969(\mathbf{2})$ & $0.912(6)$ & $0.904(\mathbf{5})$ & $0.915(\mathbf{1})$ & $0.774(4)$ & 3.4 \\
\hline $\mathrm{SC}+\mathrm{ID}$ & $0.945(\mathbf{2})$ & $0.976(\mathbf{2})$ & $0.824(\mathbf{9})$ & $0.8(6)$ & $0.775(\mathbf{1})$ & $0.969(\mathbf{2})$ & $0.914(5)$ & $0.909(\mathbf{3})$ & $0.913(\mathbf{3})$ & $0.77(7)$ & 4 \\
\hline $\mathrm{MC}+\mathrm{ID}$ & $0.941(6)$ & $0.976(2)$ & $0.831(\mathbf{6})$ & $0.811(\mathbf{1})$ & $0.768(2)$ & $0.969(\mathbf{2})$ & $0.91(8)$ & $0.903(6)$ & $0.914(\mathbf{2})$ & $0.769(\mathbf{9})$ & 4.4 \\
\hline $\mathrm{EC}+\mathrm{LL}$ & $0.945(2)$ & $0.977(\mathbf{1})$ & $0.819(\mathbf{1 1})$ & $0.808(\mathbf{2})$ & $0.707(\mathbf{9})$ & $0.968(\mathbf{8})$ & $0.918(\mathbf{2})$ & $0.911(2)$ & $0.897(\mathbf{8})$ & $0.77(7)$ & 5.2 \\
\hline $\mathrm{PEC}+\mathrm{ID}$ & $0.943(4)$ & $0.976(\mathbf{2})$ & $0.827(8)$ & $0.808(\mathbf{2})$ & $0.768(2)$ & $0.969(\mathbf{2})$ & $0.911(7)$ & $0.905(4)$ & $0.91(4)$ & $0.765(\mathbf{1 0})$ & 4.5 \\
\hline $\mathrm{PEC}+\mathrm{LL}$ & $0.944(\mathbf{3})$ & $0.977(\mathbf{1})$ & $0.819(\mathbf{1 1})$ & $0.808(2)$ & $0.707(\mathbf{9})$ & $0.968(\mathbf{8})$ & $0.911(7)$ & $0.911(2)$ & $0.896(\mathbf{9})$ & $0.771(6)$ & 5.8 \\
\hline $\mathrm{PEC}+\mathrm{LC}$ & $0.945(2)$ & $0.977(\mathbf{1})$ & $0.845(4)$ & $0.805(4)$ & $0.707(\mathbf{9})$ & $0.969(2)$ & $0.909(\mathbf{9})$ & $0.893(\mathbf{8})$ & $0.885(\mathbf{1 1})$ & $0.769(8)$ & 5.8 \\
\hline $\mathrm{PSC}+\mathrm{LL}$ & $0.94(7)$ & $0.977(\mathbf{1})$ & $0.842(\mathbf{5})$ & $0.785(\mathbf{1 1})$ & $0.759(\mathbf{3})$ & $0.968(8)$ & $0.915(4)$ & $0.896(7)$ & $0.901(\mathbf{6})$ & $0.752(\mathbf{1 1})$ & 6.3 \\
\hline $\mathrm{MC}+\mathrm{LL}$ & $0.942(\mathbf{5})$ & $0.976(\mathbf{2})$ & $0.82(\mathbf{1 0})$ & $0.808(2)$ & $0.707(\mathbf{9})$ & $0.961(\mathbf{1 5})$ & $0.916(\mathbf{3})$ & $0.904(5)$ & $0.893(\mathbf{1 0})$ & $0.769(8)$ & 6.9 \\
\hline $\mathrm{PSC}+\mathrm{ID}$ & $0.932(\mathbf{9})$ & $0.976(2)$ & $0.812(\mathbf{1 3})$ & $0.794(7)$ & $0.754(5)$ & $0.969(\mathbf{2})$ & $0.897(\mathbf{1 0})$ & $0.888(\mathbf{1 0})$ & $0.915(\mathbf{1})$ & $0.777(\mathbf{3})$ & 6.2 \\
\hline $\mathrm{EC}+\mathrm{LC}$ & $0.945(\mathbf{2})$ & $0.977(\mathbf{1})$ & $0.814(\mathbf{1 2})$ & $0.804(5)$ & $0.707(\mathbf{9})$ & $0.97(\mathbf{1})$ & $0.909(\mathbf{9})$ & $0.892(\mathbf{9})$ & $0.885(\mathbf{1 1})$ & $0.768(\mathbf{9})$ & 6.8 \\
\hline $\mathrm{SC}+\mathrm{LC}$ & $0.944(3)$ & $0.977(\mathbf{1})$ & $0.756(14)$ & $0.789(\mathbf{1 0})$ & $0.729(\mathbf{6})$ & $0.963(\mathbf{1 4})$ & $0.738(\mathbf{1 4})$ & $0.744(13)$ & $0.863(\mathbf{1 3})$ & $0.715(\mathbf{1 2})$ & 10 \\
\hline $\mathrm{PSC}+\mathrm{LC}$ & $0.94(7)$ & $0.977(\mathbf{1})$ & $0.751(\mathbf{1 5})$ & $0.806(\mathbf{3})$ & $0.722(7)$ & $0.947(\mathbf{1 6})$ & $0.763(\mathbf{1 3})$ & $0.761(\mathbf{1 2})$ & $0.742(\mathbf{1 4})$ & $0.588(\mathbf{1 3})$ & 10.1 \\
\hline RSVM & $0.948(\mathbf{1})$ & $0.977(\mathbf{1})$ & $0.851(3)$ & $0.791(\mathbf{9})$ & $0.756(4)$ & $0.965(\mathbf{1 2})$ & $0.89(\mathbf{1 2})$ & $0.874(\mathbf{1 1})$ & $0.908(\mathbf{5})$ & $0.789(\mathbf{1})$ & 5.9 \\
\hline RCNET & $0.937(\mathbf{8})$ & $0.973(\mathbf{3})$ & $0.854(\mathbf{2})$ & $0.783(\mathbf{1 2})$ & $0.709(8)$ & $0.965(\mathbf{1 2})$ & $0.894(\mathbf{1 1})$ & $0.892(\mathbf{9})$ & $0.879(\mathbf{1 2})$ & $0.773(\mathbf{5})$ & 8.2 \\
\hline
\end{tabular}

Table 5.3: Performance for the Hamming Score metric and in parenthesis the ranking of the algorithm.

The classifiers based on SPNs limiting the node splits with sequential classification (SC+LL), and SPNs with direct and indirect variable interactions using Exhaustive and Sequential classification $(\mathrm{EC}+\mathrm{ID}$ and $\mathrm{SC}+\mathrm{ID})$ obtained the best average ranking for the Hamming Score metric. In the case of Exact Match metric, the best performances were obtained by Exhaustive, Max-Prod and Pool of Exhaustive classification algorithms on SPNs with direct and indirect interactions (viz.

\footnotetext{
${ }^{4}$ Source code is available at https://github.com/nicoladimauro/dcsn.

${ }^{5}$ Available at http://meka.sourceforge.net.

${ }^{6}$ Results available at http://www.uco.es/grupos/kdis/wiki/MLdiscretization/.
} 


\begin{tabular}{|c|c|c|c|c|c|c|c|c|c|c|c|}
\hline & Arts & Business & CAL500 & Emotions & Flags & Health & Human & Plants & Scene & Yeast & Avg Rank \\
\hline $\mathrm{EC}+\mathrm{ID}$ & $0.35(\mathbf{2})$ & $0.586(\mathbf{5})$ & $0(\mathbf{1})$ & $0.361(\mathbf{6})$ & $0.125(7)$ & $0.525(\mathbf{2})$ & $0.25(4)$ & $0.236(7)$ & $0.713(\mathbf{1})$ & $0.2(2)$ & 3.7 \\
\hline $\mathrm{MC}+\mathrm{ID}$ & $0.352(\mathbf{1})$ & $0.577(8)$ & $0(\mathbf{1})$ & $0.353(7)$ & $0.125(7)$ & $0.524(3)$ & $0.256(3)$ & $0.241(\mathbf{6})$ & $0.711(2)$ & $0.2(\mathbf{2})$ & 4 \\
\hline $\mathrm{PEC}+\mathrm{ID}$ & $0.35(\mathbf{2})$ & $0.577(8)$ & $0(\mathbf{1})$ & $0.361(6)$ & $0.125(7)$ & $0.525(\mathbf{2})$ & $0.257(\mathbf{2})$ & $0.236(7)$ & $0.685(4)$ & $0.18(\mathbf{5})$ & 4.4 \\
\hline $\mathrm{PSC}+\mathrm{ID}$ & $0.277(\mathbf{1 3})$ & $0.577(8)$ & $0(\mathbf{1})$ & $0.398(\mathbf{5})$ & $0.175(\mathbf{5})$ & $0.525(2)$ & $0.206(7)$ & $0.245(5)$ & $0.711(2)$ & $0.215(\mathbf{1})$ & 4.9 \\
\hline PEC+LL & $0.311(7)$ & $0.595(\mathbf{2})$ & $0(\mathbf{1})$ & $0.415(\mathbf{1})$ & $0.205(4)$ & $0.491(\mathbf{6})$ & $0.257(2)$ & $0.122(\mathbf{1 1})$ & $0.591(\mathbf{9})$ & $0.165(6)$ & 4.9 \\
\hline $\mathrm{SC}+\mathrm{ID}$ & $0.329(\mathbf{3})$ & $0.583(7)$ & $0(\mathbf{1})$ & $0.336(8)$ & $0.125(7)$ & $0.516(4)$ & $0.241(5)$ & $0.204(\mathbf{9})$ & $0.705(3)$ & $0.182(4)$ & 5.1 \\
\hline $\mathrm{EC}+\mathrm{LC}$ & $0.297(\mathbf{1 1})$ & $0.591(4)$ & $0(\mathbf{1})$ & $0.407(\mathbf{2})$ & $0.205(4)$ & $0.514(5)$ & $0.196(8)$ & $0.31(\mathbf{2})$ & $0.591(\mathbf{9})$ & $0.184(\mathbf{3})$ & 4.9 \\
\hline $\mathrm{PSC}+\mathrm{LL}$ & $0.315(5)$ & $0.595(2)$ & $0(\mathbf{1})$ & $0.297(\mathbf{1 0})$ & $0.237(\mathbf{1})$ & $0.474(8)$ & $0.215(6)$ & $0.298(\mathbf{3})$ & $0.618(6)$ & $0.122(\mathbf{1 2})$ & 5.4 \\
\hline $\mathrm{PEC}+\mathrm{LC}$ & $0.297(\mathbf{1 1})$ & $0.591(4)$ & $0(\mathbf{1})$ & $0.407(\mathbf{2})$ & $0.205(4)$ & $0.514(\mathbf{5})$ & $0.196(8)$ & $0.31(\mathbf{2})$ & $0.554(\mathbf{1 1})$ & $0.182(4)$ & 5.2 \\
\hline $\mathrm{SC}+\mathrm{LL}$ & $0.3(\mathbf{9})$ & $0.596(\mathbf{1})$ & $0(\mathbf{1})$ & $0.294(\mathbf{1 2})$ & $0.205(4)$ & $0.6(\mathbf{1})$ & $0.147(\mathbf{1 0})$ & $0.215(8)$ & $0.593(\mathbf{8})$ & $0.124(\mathbf{1 1})$ & 6.5 \\
\hline $\mathrm{EC}+\mathrm{LL}$ & $0.299(\mathbf{1 0})$ & $0.595(2)$ & $0(\mathbf{1})$ & $0.415(\mathbf{1})$ & $0.205(4)$ & $0.49(7)$ & $0.121(\mathbf{1 2})$ & $0.122(\mathbf{1 2})$ & $0.598(7)$ & $0.163(7)$ & 6.3 \\
\hline $\mathrm{MC}+\mathrm{LL}$ & $0.325(4)$ & $0.576(\mathbf{9})$ & $0(\mathbf{1})$ & $0.407(2)$ & $0.205(4)$ & $0.363(\mathbf{1 2})$ & $0.135(\mathbf{1 1})$ & $0.282(4)$ & $0.589(\mathbf{1 0})$ & $0.157(\mathbf{8})$ & 6.5 \\
\hline $\mathrm{PSC}+\mathrm{LC}$ & $0.307(8)$ & $0.592(3)$ & $0(\mathbf{1})$ & $0.407(\mathbf{2})$ & $0.231(\mathbf{2})$ & $0.402(\mathbf{1 0})$ & $0.018(\mathbf{1 4})$ & $0(\mathbf{1 4})$ & $0.187(\mathbf{1 4})$ & $0.008(\mathbf{1 4})$ & 8.2 \\
\hline $\mathrm{SC}+\mathrm{LC}$ & $0.289(\mathbf{1 2})$ & $0.592(\mathbf{3})$ & $0(\mathbf{1})$ & $0.361(\mathbf{6})$ & $0.205(4)$ & $0.456(\mathbf{9})$ & $0.02(\mathbf{1 3})$ & $0.067(\mathbf{1 3})$ & $0.442(\mathbf{1 3})$ & $0.072(\mathbf{1 3})$ & 8.7 \\
\hline RSVM & $0.226(\mathbf{1 4})$ & $0.584(6)$ & $0(\mathbf{1})$ & $0.303(\mathbf{9})$ & $0.211(\mathbf{3})$ & $0.393(\mathbf{1 1})$ & $0.185(\mathbf{9})$ & $0.195(\mathbf{1 0})$ & $0.64(5)$ & $0.155(\mathbf{9})$ & 7.7 \\
\hline RCNET & $0.313(6)$ & $0.567(\mathbf{1 0})$ & $0(\mathbf{1})$ & $0.295(\mathbf{1 1})$ & $0.17(6)$ & $0.456(\mathbf{9})$ & $0.263(\mathbf{1})$ & $0.344(\mathbf{1})$ & $0.554(\mathbf{1 2})$ & $0.147(\mathbf{1 0})$ & 6.7 \\
\hline
\end{tabular}

Table 5.4: Performance for the Exact Match metric and in parenthesis the ranking of the algorithm.

\begin{tabular}{|c|c|c|c|c|c|c|c|c|c|c|c|}
\hline & Arts & Business & CAL500 & Emotions & Flags & Health & Human & Plants & Scene & Yeast & AvgRank \\
\hline $\mathrm{PSC}+\mathrm{ID}$ & $0.404(6)$ & $0.722(\mathbf{9})$ & $0.351(\mathbf{1})$ & $0.609(5)$ & $0.65(\mathbf{1})$ & $0.634(\mathbf{1})$ & $0.336(\mathbf{2})$ & $0.302(5)$ & $0.742(\mathbf{2})$ & $0.502(3)$ & 3.5 \\
\hline $\mathrm{PSC}+\mathrm{LL}$ & $0.425(4)$ & $0.741(\mathbf{1})$ & $0.319(\mathbf{3})$ & $0.615(2)$ & $0.621(6)$ & $0.625(4)$ & $0.257(\mathbf{5})$ & $0.327(\mathbf{3})$ & $0.702(6)$ & $0.511(2)$ & 3.6 \\
\hline $\mathrm{PEC}+\mathrm{ID}$ & $0.426(\mathbf{3})$ & $0.739(\mathbf{2})$ & $0.219(\mathbf{1 1})$ & $0.607(6)$ & $0.626(3)$ & $0.634(\mathbf{1})$ & $0.293(\mathbf{3})$ & $0.241(8)$ & $0.717(5)$ & $0.5(4)$ & 4.6 \\
\hline $\mathrm{EC}+\mathrm{ID}$ & $0.426(3)$ & $0.738(\mathbf{3})$ & $0.216(\mathbf{1 2})$ & $0.607(6)$ & $0.626(3)$ & $0.634(\mathbf{1})$ & $0.28(5)$ & $0.241(8)$ & $0.744(\mathbf{1})$ & $0.499(5)$ & 4.7 \\
\hline $\mathrm{MC}+\mathrm{ID}$ & $0.429(2)$ & $0.733(6)$ & $0.214(\mathbf{1 3})$ & $0.592(7)$ & $0.623(\mathbf{5})$ & $0.632(\mathbf{2})$ & $0.289(4)$ & $0.246(7)$ & $0.742(2)$ & $0.497(6)$ & 5.4 \\
\hline $\mathrm{SC}+\mathrm{ID}$ & $0.402(7)$ & $0.734(5)$ & $0.327(\mathbf{2})$ & $0.591(8)$ & $0.638(2)$ & $0.626(3)$ & $0.241(6)$ & $0.214(\mathbf{1 1})$ & $0.737(\mathbf{3})$ & $0.483(\mathbf{9})$ & 5.6 \\
\hline $\mathrm{EC}+\mathrm{LC}$ & $0.368(\mathbf{1 0})$ & $0.739(\mathbf{2})$ & $0.225(\mathbf{1 0})$ & $0.611(4)$ & $0.58(\mathbf{9})$ & $0.634(\mathbf{1})$ & $0.224(8)$ & $0.325(4)$ & $0.638(8)$ & $0.491(7)$ & 6.3 \\
\hline $\mathrm{PEC}+\mathrm{LC}$ & $0.368(\mathbf{1 0})$ & $0.739(2)$ & $0.249(7)$ & $0.607(8)$ & $0.58(\mathbf{9})$ & $0.634(\mathbf{1})$ & $0.224(8)$ & $0.325(4)$ & $0.628(\mathbf{1 0})$ & $0.487(8)$ & 6.7 \\
\hline $\mathrm{PEC}+\mathrm{LL}$ & $0.37(\mathbf{9})$ & $0.739(\mathbf{2})$ & $0.229(8)$ & $0.609(5)$ & $0.58(\mathbf{9})$ & $0.61(5)$ & $0.293(\mathbf{3})$ & $0.124(\mathbf{1 2})$ & $0.624(\mathbf{1 1})$ & $0.481(\mathbf{1 0})$ & 7.4 \\
\hline $\mathrm{PSC}+\mathrm{LC}$ & $0.417(5)$ & $0.737(4)$ & $0.279(4)$ & $0.615(2)$ & $0.604(7)$ & $0.557(\mathbf{1 0})$ & $0.227(7)$ & $0.218(\mathbf{1 0})$ & $0.474(13)$ & $0.403(\mathbf{1 6})$ & 7.8 \\
\hline $\mathrm{EC}+\mathrm{LL}$ & $0.367(\mathbf{1 1})$ & $0.738(\mathbf{3})$ & $0.229(8)$ & $0.618(\mathbf{1})$ & $0.58(\mathbf{9})$ & $0.61(5)$ & $0.136(\mathbf{1 2})$ & $0.124(\mathbf{1 2})$ & $0.631(\mathbf{9})$ & $0.48(11)$ & 8.1 \\
\hline $\mathrm{SC}+\mathrm{LL}$ & $0.359(\mathbf{1 2})$ & $0.741(\mathbf{1})$ & $0.276(5)$ & $0.581(\mathbf{9})$ & $0.594(8)$ & $0.6(7)$ & $0.166(\mathbf{1 0})$ & $0.218(\mathbf{1 0})$ & $0.628(\mathbf{1 0})$ & $0.476(\mathbf{1 3})$ & 8.5 \\
\hline $\mathrm{SC}+\mathrm{LC}$ & $0.352(\mathbf{1 3})$ & $0.738(3)$ & $0.263(6)$ & $0.592(7)$ & $0.594(8)$ & $0.581(8)$ & $0.216(\mathbf{9})$ & $0.224(\mathbf{9})$ & $0.484(\mathbf{1 2})$ & $0.427(\mathbf{1 5})$ & 9 \\
\hline $\mathrm{MC}+\mathrm{LL}$ & $0.396(8)$ & $0.729(7)$ & $0.226(\mathbf{9})$ & $0.613(3)$ & $0.58(\mathbf{9})$ & $0.484(\mathbf{1 1})$ & $0.154(\mathbf{1 1})$ & $0.285(6)$ & $0.624(\mathbf{1 1})$ & $0.477(12)$ & 8.7 \\
\hline RSVM & $0.304(\mathbf{1 4})$ & $0.738(\mathbf{3})$ & $0.002(\mathbf{1 5})$ & $0.561(\mathbf{1 0})$ & $0.624(4)$ & $0.569(\mathbf{9})$ & $0.293(\mathbf{3})$ & $0.33(\mathbf{2})$ & $0.736(4)$ & $0.526(\mathbf{1})$ & 6.5 \\
\hline RCNET & $0.432(\mathbf{1})$ & $0.728(8)$ & $0.203(14)$ & $0.554(\mathbf{1 1})$ & $0.563(\mathbf{1 0})$ & $0.603(6)$ & $0.361(\mathbf{1})$ & $0.397(\mathbf{1})$ & $0.669(7)$ & $0.464(\mathbf{1 4})$ & 7.3 \\
\hline
\end{tabular}

Table 5.5: Performance for the Accuracy metric and in parenthesis the ranking of the algorithm.

$\mathrm{EC}+\mathrm{ID}, \mathrm{MC}+\mathrm{ID}$, and PEC $+\mathrm{ID})$. Finally, the results for Accuracy metric show that Pool of Sequential classification on SPNs limiting node splits, Pools of Sequential and Exhaustive classifications on SPNs with direct and indirect variables interactions obtained highest performance (viz. PSC $+\mathrm{LL}$, $\mathrm{PEC}+\mathrm{ID}$ and PSC+ID).

These results reveal that in general, the proposed classifiers based on SPNs that use the learning algorithm with direct and indirect variables interactions (ID) outperforms the classifiers that learn with limit node splits (LL) or uses multivariate distributions as leaves (LC). Although, the complexity time of ID is higher compared with LL and LC, because it simultaneously learns two structures (SPN and Markov network). For the classifiers using Sequential classification, on average the best label ordering criteria are Static Best-First and Random. While using Pool classification the best criteria for combining the initial set of classifications are Weighted average and Majority voting.

According to our results, the best-proposed Multi-label classifiers ranks better than the stateof-art Multi-label classifiers evaluated in terms of Exact Match, Hamming Score, and Accuracy. RCNET ranks above 6 on average for all metrics. Finding the optimal classification (MPE) usually improved accuracy, especially with respect to Accuracy. This motivates the development of better algorithms for MPE in SPNs and suggests that SPNs are indeed competitive when exact inference is performed. RCNET only outperforms, in terms of Accuracy metric, our proposed classifier using Sum-Product Networks in three datasets (Arts, Human, and Plants).

The RaKel approach with Support Vector machines as basis multi-class classifier (RSVM) ranked above 5 on average for all metrics, and only shows better results for Accuracy metric in the datasets from the Biological domain (Human, Plants, and Yeast). While the proposed classifiers obtained better results in the others dataset, especially on Text, Music and Image domains where outper- 
forms the state-of-art classifiers.

We will focus our discussion on the results obtained for Accuracy (see Table 5.5), as presented in previous sections it is one of the best measures for MLC. The proposed Multi-Label classifiers based on Sum-Product networks did not show the best performance for Plants, Human, Arts and Yeast dataset; according to our analysis, these datasets do not have any common characteristic, except that some of them have low label cardinality. In the other hand, our Multi-Label classifiers perform better in datasets with a large number of labels and a low number of training examples (viz. CAL500, Flags), in addition to the best results for Business, Emotions, Health and Scene.

\subsubsection{Comparing classifiers with Wilcoxon signed-rank test}

The Wilcoxon signed-rank test is a non-parametric statistical hypothesis test (null hypothesis significance test) used when comparing two paired samples [Dem06]. Then, it can be used to compare the performance of two classifiers on a large collection of datasets (greater than ten datasets) for a specific metric. Given a significance level $\alpha$ (normally set to $\alpha=0.05$ ), if the result of the test, p-value, is less than $\alpha$, indicates that a classifier has a statistically significant difference with the other, otherwise that is not enough evidence to assume any conclusion.

We use the Wilcoxon signed-rank test to compare RSVM and the performance of our best performed classifier for each metric, according with the Tables 5.3, 5.4 and 5.5.

\begin{tabular}{lcc}
\hline Metric & Compared Classifiers & p-value \\
\hline Hamming Score & SC+LL vs RSMV & 0.6833 \\
Exact Match & EC+ID vs RSVM & 0.0469 \\
Accuracy & PSC + ID vs RSVM & 0.0745 \\
\hline
\end{tabular}

Table 5.6: P-value obtained from the Wilcoxon signed-rank test on the Compared classifiers for each Metric

The results obtained for the classifiers comparisons of Hamming Score and Accuracy metrics, indicating that is not enough evidence to affirm that the compared classifiers have statistically significant difference. Therefore, the obtained results are not conclusive and may have been affected by the number of dataset considered in our experiments (10 datasets).

\subsubsection{Comparing classifiers with Bayesian signed-rank test}

Bayesian tests can accept the null hypotheses, calculating the posterior probability of the null and the alternative hypotheses. While the null-hypothesis significance tests can only reject the null hypothesis. The Bayesian signed-rank test returns probabilities that, based on the measured performance, one classifier is better than another or vice versa or they are within the region of practical equivalence (rope) [BMC $\left.{ }^{+} 14\right]$, [BCDZ17].

Figures 5.1, 5.2 and 5.3 shows the results obtained for the Bayesian signed-rank test (Considering rope $=0.01$ ) on pairwise evaluations for RSVM, RCNET and our proposed classifiers for HS, EM and Acc respectively (According with the Tables 5.3, 5.4 and 5.5). Darker cells represent greater probability of "row classifier" being better than "column classifier", this probability is also show on the cell. For example, in the Figure 5.1, $P(\mathrm{PSC}+\mathrm{ID}>\mathrm{RSVM})=0.898$ (dark blue), $P(\mathrm{RSVM}$ $>\mathrm{PSC}+\mathrm{ID})=0.102$ (light blue) and $P(\mathrm{PSC}+\mathrm{ID}$ rope $\mathrm{RSVM})=1-0.898-0.0102=0$ (not showed in the figure). Note that, the probability of the best rank classifiers based on SPN been better than the state-of-art (RSVM and RCNET) is highest, consequently these cells are the darkest. 


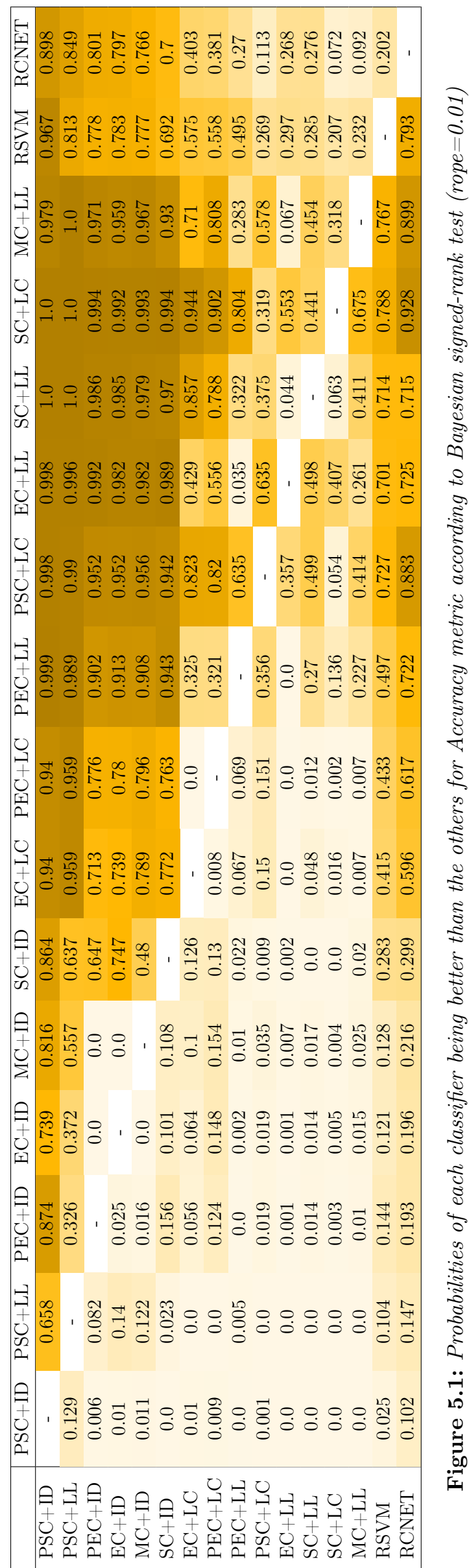




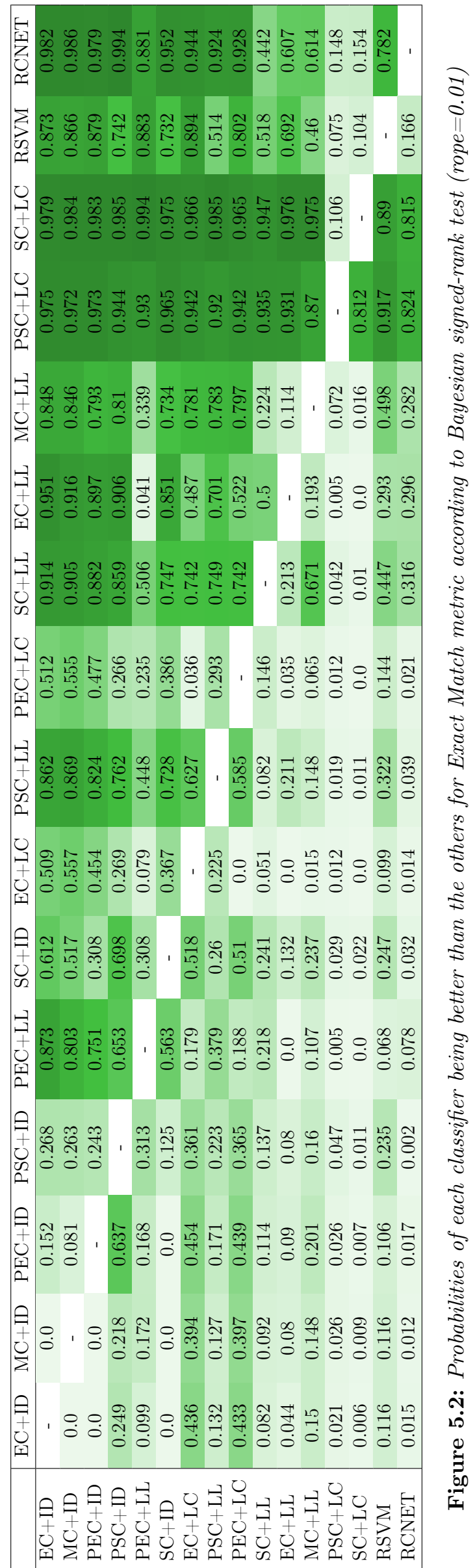




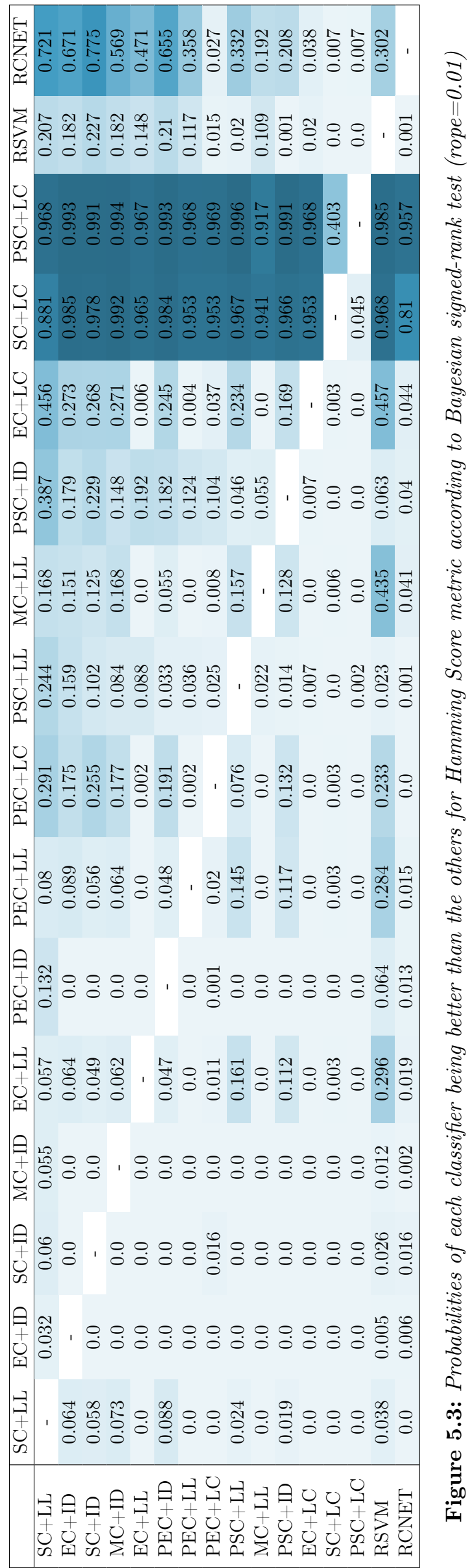


In addition, the Figure 5.4 reports the samples from the posteriors (represents as points), the simplex (the large orange triangle) and three regions (in orange). For instance, the region at the bottom-left of the triangle is relative to the case where "left classifier" is more probable than "rope" and "right classifier" together; the region at the top of the triangle represents the case where "rope" is more probable and the region bottom-left the "right classifier" is more probable.

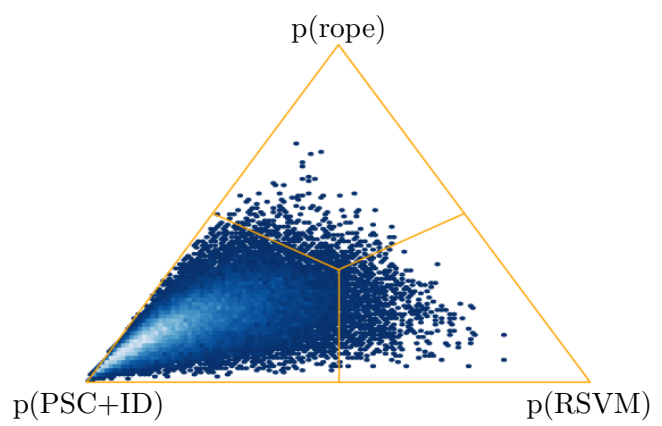

(a)

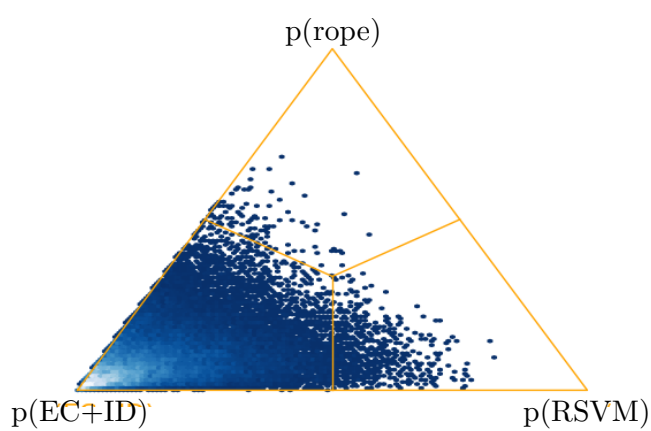

(b)

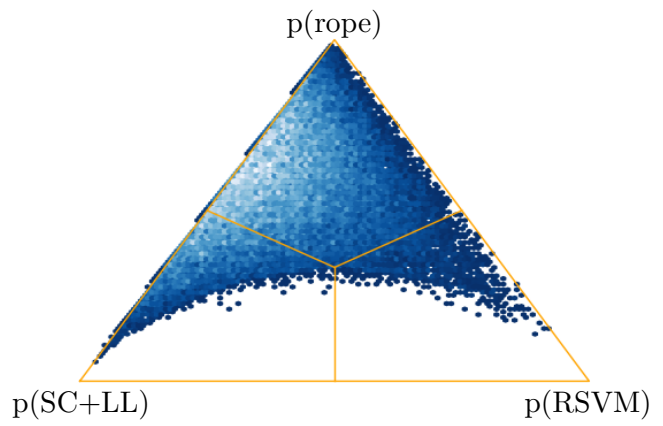

(c)

Figure 5.4: (a) Reports the posterior for PSC+ID vs. RVSM for Accuracy Metric, (b) Reports the posterior for EC+ID vs. RVSM for Exact Match Metric and (c) Reports the posterior for $S C+L L$ vs. RVSM for Hamming Score Metric for the Bayesian sign-rank test (rope=0.01).

For the three posterior at the Figure 5.4, the probabilities for the "left classifiers" (based on SPN) been better than the "right classifier" (RSVM) is greater than the opposite case. In the case of Hamming Score metric, the posterior shows that the classifiers are practical equivalents or SC+LL is better than RSVM with hight probability. 


\section{Chapter 6}

\section{Conclusions}

Multi-Label Classification (MLC) generalize traditional classification by associating every example with a set of relevant labels. MLC has been shown to be much more challenging than traditional (i.e., single-label) classification. In this work, we investigated the use of Sum-Product Networks (SPN) for Multi-Label Classification. We exploited several approaches varying either the algorithm used for learning the network or the algorithm used for issuing classifications. We compared our proposed approaches against two state-of-the-art Multi-Label classifiers on a set of benchmark datasets.

The results showed that our approaches are competitive of the state-of-the-art methods (viz. RCNET and Rakel with SVM). The proposed classifiers based on Sum-Product Networks outperformed the other classifiers in terms of Accuracy, Hamming Score and Exact Match metrics. Especially a dataset with a very high number of relevant labels, our approaches clearly outperformed the other approaches. This suggests that SPNs might better capture the intricate dependences among labels, which might be crucial in domains with a high number of interdependent labels. Although,further experiments are need to verify this claim.

Learning more expressive SPNs using the Indirect and Direct learning algorithm [RL14] resulted in highest accuracy, but also in longest learning time, which might be a problem for domains with very large number of labels. SPN classifiers learned using the method described in [VDME15] performed slightly worse than those learned using the ID algorithm with much smaller training times.

If the main concern is the model learning time, we recommend use Pool of Sequential classification on SPNs limiting node splits, which average rank was five for the three evaluated metrics. While if the main concern is the classification time, the Max-Prod classification on SPN with direct and indirect variable interactions is the best option, that is the second best average rank.

\subsection{Suggestions for Future Research}

In future work, we intent to investigate the use SPNs within the Random k-Label sets (RaKel) approach. Propose new algorithms for approximate the Most Probable Explanation inspired on several approach for Multi-Label Classification, such as Hierarchy Of Multi-Label classifiERs (HOMER) and RaKel.

Also, would like to investigate the performance of the proposed classifiers using another methods for learning an Sum-Product Networks. 


\section{Bibliography}

[ABG15] Tameem Adel, David Balduzzi and Ali Ghodsi. Learning the structure of sum-product networks via an SVD-based algorithm. In Proceedings of The Conference on Uncertainty in Artificial Intelligence, 2015. 1

[ACMG13] Antonucci Alessandro, Giorgio Corani, Denis Mauá and Sandra Gabaglio. An ensemble of Bayesian networks for multi-label classification. In Proceedings of the 23th International Joint Conference on Artificial Intelligence, pages 1220-1225, 2013. 1, 17

[BCDZ17] Alessio Benavoli, Giorgio Corani, Janez Demšar and Marco Zaffalon. Time for a change: a tutorial for comparing multiple classifiers through bayesian analysis. Journal of $M a$ chine Learning Research, pages 1-36, 2017. 34

[BLSB04] Matthew R Boutell, Jiebo Luo, Xipeng Shen and Christopher M Brown. Learning multilabel scene classification. International Journal of Pattern Recognition, 37(9):17571771, 2004. 1, 30

$\left[\mathrm{BMC}^{+} 14\right]$ A. Benavoli, F. Mangili, G. Corani, M. Zaffalon and F. Ruggeri. A Bayesian Wilcoxon signed-rank test based on the Dirichlet process. In Proceedings of the 30th International Conference on Machine Learning (ICML 2014), pages 1-9, 2014. 34

[CAMG14] Giorgio Corani, Alessandro Antonucci, Denis D Mauá and Sandra Gabaglio. Trading off speed and accuracy in multi-label classification. In Proceedings of the European Workshop on Probabilistic Graphical Models, pages 145-159, 2014. 1, 17

[CK01] Amanda Clare and Ross D King. Knowledge discovery in multi-label phenotype data. In Proceedings of European Conference on Principles of Data Mining and Knowledge Discovery, pages 42-53, 2001. 1

[CL68] C. Chow and C. Liu. Approximating discrete probability distributions with dependence trees. IEEE Transactions on Information Theory, 14(3):462-467, 1968. 3

[CLGV16] Alberto Cano, José María Luna, Eva L Gibaja and Sebastián Ventura. LAIM discretization for multi-label data. International Journal Information Sciences, 330:370-384, 2016. 29,30

[CMM11] Everton Alvares Cherman, Maria Carolina Monard and Jean Metz. Multi-label problem transformation methods: A case study. Electronic Journal CLEI, 14(1):4-4, 2011. 16, 17

[Dem06] Janez Demšar. Statistical comparisons of classifiers over multiple data sets. Journal of Machine learning Research, pages 1-30, 2006. 34

[DMVE16] Nicola Di Mauro, Antonio Vergari and Floriana Esposito. Multi-label classification with cutset networks. In Proceedings of the 8th International Conference on Probabilistic Graphical Models, pages 147-158, 2016. 29, 30, 32 
[DV12] Aaron Dennis and Dan Ventura. Learning the architecture of sum-product networks using clustering on variables. In Proceedings of Advances in Neural Information Processing Systems, pages 2033-2041, 2012. 6

[DWCH12] Krzysztof Dembczyński, Willem Waegeman, Weiwei Cheng and Eyke Hüllermeier. On label dependence and loss minimization in multi-label classification. International Journal of Machine Learning, 88(1-2):5-45, 2012. 1, 30

[EW02] André Elisseeff and Jason Weston. A kernel method for multi-labelled classification. In Proceedings of the Advances in Neural Information Processing Systems, pages 681-687, 2002. 30

[FGG97] Nir Friedman, Dan Geiger and Moises Goldszmidt. Bayesian network classifiers. International Journal of Machine learning, 29(2-3):131-163, 1997. 1, 17

[GD12] Robert Gens and Pedro Domingos. Discriminative learning of sum-product networks. In Proceedings of the Advances in Neural Information Processing Systems, pages 32483256, 2012. 1

[GD13] Robert Gens and Pedro M Domingos. Learning the structure of sum-product networks. In Proceedings of the International Conference on Machine Learning, pages 873-880, 2013. 6

[KF09] Daphne Koller and Nir Friedman. Probabilistic graphical models: principles and techniques. MIT press, 2009. 17

[LI13] Qiang Liu and Alexander Ihler. Variational algorithms for marginal map. The Journal of Machine Learning Research, 14(1):3165-3200, 2013. 20

[LM17] Julissa Villanueva Llerena and Denis Deratani Mauá. On using sum-product networks for multi-label classification. In Proceedings of the 6th Brazilian Conference on Intelligent Systems, BRACIS, pages 25-30, 2017. 2

[LMP01] John Lafferty, Andrew McCallum and Fernando Pereira. Conditional random fields: Probabilistic models for segmenting and labeling sequence data. In Proceedings of the 8th International Conference on Machine Learning, volume 1, pages 282-289, 2001. 1, 17

[LR13] Daniel Lowd and Amirmohammad Rooshenas. Learning markov networks with arithmetic circuits. In Proceedings of the International Conference on Artificial Intelligence and Statistics, pages 406-414, 2013. 11

[LR15] Daniel Lowd and Amirmohammad Rooshenas. The Libra toolkit for probabilistic models. Journal of Machine Learning Research, 16:2459-2463, 2015. 31

[PD11] Hoifung Poon and Pedro Domingos. Sum-product networks: A new deep architecture. In Proceedings of the 27th Conference on Uncertainty in Artificial Intelligence, pages 337-346, 2011. 1, 2, 3, 5, 6, 21

[PGP13] Robert Peharz, Bernhard C Geiger and Franz Pernkopf. Greedy part-wise learning of sum-product networks. In Proceedings of the Joint European Conference on Machine Learning and Knowledge Discovery in Databases, pages 612-627, 2013. 6

[PGPD16] R. Peharz, R. Gens, F. Pernkopf and P. Domingos. On the latent variable interpretation in sum-product networks. Journal of Transactions on Pattern Analysis and Machine Intelligence, pages 1-14, 2016. 2, 5 
[RL14] Amirmohammad Rooshenas and Daniel Lowd. Learning sum-product networks with direct and indirect variable interactions. In Proceedings of the International Conference on Machine Learning, pages 710-718, 2014. 12, 39

[RPHF11] Jesse Read, Bernhard Pfahringer, Geoff Holmes and Eibe Frank. Classifier chains for multi-label classification. International Journal of Machine learning, 85(3):333-359, 2011. 1, 16, 23

[SC16] B. M. Sguerra and F. G. Cozman. Image classification using sum-product networks for autonomous flight of micro aerial vehicles. In Proceeding of the 5th Brazilian Conference on Intelligent Systems, pages 139-144, 2016. 1

[TBTL08] Douglas Turnbull, Luke Barrington, David Torres and Gert Lanckriet. Semantic annotation and retrieval of music and sound effects. IEEE Transactions on Audio, Speech, and Language Processing, 16(2):467-476, 2008. 29

[TK06] Grigorios Tsoumakas and Ioannis Katakis. Multi-label classification: An overview. International Journal of Data Warehousing and Mining, 3(3), 2006. 1

[TKV11] Grigorios Tsoumakas, Ioannis Katakis and Ioannis Vlahavas. Random k-labelsets for multi-label classification. IEEE Transactions on Knowledge and Data Engineering, 23(7):1079-1089, 2011. 32

[TTKV08] Konstantinos Trohidis, Grigorios Tsoumakas, George Kalliris and Ioannis P Vlahavas. Multi-label classification of music into emotions. In Proceedings of International Symposium on Music Information Retrieval, volume 8, pages 325-330, 2008. 1, 30

[US03] Naonori Ueda and Kazumi Saito. Parametric mixture models for multi-labeled text. In Proceedings of the Advances in Neural Information Processing Systems, pages 737-744, 2003. 29

[VDME15] Antonio Vergari, Nicola Di Mauro and Floriana Esposito. Simplifying, regularizing and strengthening sum-product network structure learning. In Proceedings of the Joint European Conference on Machine Learning and Knowledge Discovery in Databases, pages 343-358, 2015. 8, 9, 39 